\title{
Modern contraceptive use in Northwestern Region of Nigeria: Rural-Urban segmentation analysis
}

\author{
Muyiwa Oladosun', Tayo O. George ${ }^{1}$, Anthony Onwumah ${ }^{1}$, \\ Adebanke Olawole-Isaac ${ }^{1}$, \& Dorcas O. Adekoya ${ }^{2}$ \\ ${ }^{1}$ Public Private Partnership Research Cluster, Department of Economics \\ and Development Studies Covenant University, Canaan land, \\ Ota, Ogun State, Nigeria \\ ${ }^{2}$ Independent Researcher, Ota, Ogun State, Nigeria \\ Email: muyiwa.oladosun@covenantuniversity.edu.ng
}

\begin{abstract}
Background: Northwestern region of Nigeria has among the lowest modern contraceptive use, and the highest fertility rate in the country, whereas there are little or no disaggregated information at the ruralurban level to shed light on the reasons for low use.

Method: We analysed the 2013 Nigeria Demographic and Health Survey. The relationships between modern contraceptive use and predictors were examined using Chi-square and logistics regression.

Findings: Findings showed that common determinants of modern contraceptive use among rural and urban respondents were, state of residence, religion, and fertility preference. Determinants unique to rural respondents were; education, wealth status, number of siblings of respondents, and household had cable TV, and for urban respondents, unique determinants were, birth order of respondents, husband/partner's education, household had radio, and mobile phone.

Conclusion: Policy and program interventions need to use these additional insights to increase uptake of modern contraceptive use in the region.
\end{abstract}

Keywords: Modern Contraceptive Use, Rural-Urban Segmentation, North-Western Region, Agents of Modernity, Fertility Behavior

\section{Introduction}

Between 2019 and 2050, Nigeria's population growth rate is expected to be second to that of India (United Nations, 2019). With India currently ranked the second most populous country in the world, Nigeria's growth rate suggest huge inertia for future fast population increases. Nigeria is currently ranked the seventh largest population in the world (20I million), and is expected to become the third largest (40I.3 million) by 2050 (United Nations, 2019). The inertia for fast population growth in Nigeria must be slowed through concerted effort from all stakeholders especially considering the current poor poverty index and slow infrastructural development in the country.

In recent times, a number of studies have attempted to investigate the socio-demographic characteristics influencing fertility preference and contraceptive use across Nigeria (Babalola et al, 2015; Ibisomi, 20l4; Asekun-Olarinmoye et al, 2013; Olalekan and Olufunmilayo, 20 I2; Igbodekwe et al, 2008). These studies suggest that considerable gains are possible in the understanding of the determinants of contraceptive behavior by providing more insights on the key predictors influencing contraceptive decisions.
These suggestions are against the backdrop of plateaued-like state of high fertility and persistent low family planning acceptance and modern contraceptive use in the country. Statistics from the 2003 Nigeria Demographic and Health Survey (NDHS) showed that Total Fertility Rate (TFR) was 5.7 and modern contraceptive use was $8.2 \%$ (NPC [Nigeria] and ORC Macro, 2004), and the 2013 NDHS show only slight improvement, after 10 years of program intervention, with TFR of 5.5 (reduction of 0.2 ) and modern contraceptive use of $9.8 \%$ (increase of I.6\%).

Another major characteristic of the fertility and modern contraception relationship is significant consistent residential and regional dichotomy over the years. In 2003 the TFR for rural areas was more than that of the urban areas (6.1 and 4.9 respectively), and in 2013 the gap remains (6.2 and 4.7 respectively). Similarly, modern contraceptive use has been consistently lower in rural than in urban areas. In 2003 modern contraceptive use was 5.7\% in rural, and $13.9 \%$ in urban, and in 2013 the figures were $5.7 \%$ and $16 \%$ respectively. Another characteristic that can provide depth of information 
on the fertility and modern contraception relationships is regional statistical variations. The 2003 NDHS results showed that northwestern region, the focus of this study, has the second highest fertility rate in the country (6.7), the highest in 2013 NDHS. Conversely, modern contraceptive use was second lowest for the region in both 2003 (3.3\%) and 2013 (3.6\%) (NPC [Nigeria] and ORC Macro, 2004; NPC [Nigeria] and ICF International, 20 I3).

Bridging the gaps in regional and residential variations in fertility and modern contraceptive use will substantially reduce fertility, and increase overall contraceptive uptake in the country. This will require context specific information that will inform customized more effective programming geared to meet specific needs of sub-groups. Persistent high fertility and low modern contraceptive use calls for more insights on the dynamics of fertility behavior and contraceptive use in the country and especially on the region of focus to achieve substantial results. This study applied segmentation theory principles to understand fertility dynamics and determinants of modern contraceptive use in the northwestern region of Nigeria. It fills the gaps in rural-urban differentials in key determinants of contraceptive use in the region with a view to providing more customtailored information for effective targeting, and programming on family planning.

\section{Literature review \& conceptualization}

This section discusses evidence in the literature on the relationships between known key determinants of modern contraception starting with the background factors. Evidence suggest that education has a positive relationship with contraceptive uptake. Nigeria women who have attained a tertiary education are more likely to have ever used modern contraceptives compared to those who had secondary education or less (Babalola et al, 2015; lgbodekwe et al, 2008; Olalekan and Olufunmilayo, 20/2). Studies have established a high level of awareness of any method of contraceptive use among women of childbearing age in Nigeria (85\%) but very low level of use (NPC [Nigeria] and ICF International, 20I3). Further analysis into modern contraceptive use suggest wide variations across women of childbearing age in Northwestern Nigeria. Although 76 percent of the women were aware of any method (traditional \& modern) contraception, only 3 percent of them had ever used modern contraceptives. Insights into state level variations show that across the states of the nation, modern contraceptive use ranges from 30 percent in Osun southwestern Nigeria to less than I percent in Jigawa and Kano state Northwestern Nigeria (NPC [Nigeria] and ICF International, 20/3).
Family preferences and contraceptive use remains one of the most important reproductive health decisions that couples have to make (Owoloko and Oladosun, 2018; Gertner, 1990). Modern contraceptive use is often adopted by couples who have no preference for additional children or wish to limit the number of children they want to have in addition to controlling the spacing of children (Oladosun et al, 2019; Olaitan, 20I I). Family planning measures are designed specifically to regulate the number of children and spacing of children within a family, for the purpose of population growth reduction in addition to ensuring the quality of life. A person's social; environment among other factors has been found to influence family planning decisions. People choose contraceptive methods that are commonly used in their community because of the belief that it is socially acceptable to do so (Ouma et al, 2016; Gueye et al, 2015; Rogers and Kincaid, 2000; Valente and Thomas, 200I). In general, community norms i.e. gender roles, social networks, religion and local beliefs determine individual childbearing practices and sexual and reproductive behavior (Ochako et al, 2015; Bosveld, 1998). The entire community may be encouraged to use one type of contraceptive rather than choice based on individual needs (Ochako et al, 20 I5; Potter, 1999).

In northwestern region of Nigeria, key factors influencing the choice of family planning effectiveness identified in the literature are religion, cost, health risk, preference, and gender role (Zaggi, 2014; Odewale et al, 2016; Olaitan, 20I I).

Despite consistent variations established in past NDHS reports, there are no information on the dynamics, and determinants of modern contraception at the regional level in the country. Most available literature deal with state specific contraceptive behavior whereas knowledge at the regional level, examining each region as a group are also vital for planning and program implementation purposes. Aspects of these gaps in the literature is what this paper address in the Northwestern region which in 2016 was estimated to house about 48.9 million people, over $25.3 \%$ of the entire population of the country (National Bureau of Statistics [NBS], 20/8). The region is unique because its 2016 total fertility rate of 7.3 was by far higher than that of the national average of 5.8 with Jigawa state (8.5) recording the highest in the country. It is also important to note that none of the seven states in the region (except Kaduna state) recorded more than 5\% modern contraception, about half of the national average of $10.8 \%$ with Jigawa state recording the lowest of I.1\% (NBS, 2018). These demographic scenarios make the northwestern region one of the largest 
contributors to population increase in the country and the key rationale for focusing on the region.

For Nigeria to achieve its demographic dividend, with the aim of eradicating poverty, improve quality of life of citizens, economic growth, health and wellbeing of families and societies, there is the need to improve information on the dynamics between fertility and modern contraceptive use, and sociodemographic characteristics of women of childbearing age in Northwestern Nigeria.

In this study, modern contraceptive use is conceptualized to be directly and indirectly influenced by key predictors. Background factors such as age, state of residence, education, religion, wealth status, number of co-wives, living arrangement, husband/partner's age, husband/partner's education, and husband/partner's type of work are assumed to have direct relationships with modern contraceptive use. While respondent's number of siblings, respondent's birth order, employment status, type of employment, and earnings from work are assumed to have indirect relationships with contraceptive use working through fertility behavior. Agents of modernity such as household possession of electricity, radio, TV, cable TV, mobile phone and exposure to thee agents have direct relatonships with modern contraception. Fertility behavior measured as number of living children, and preference to have another child is assumed to have direct relationship with modern contraception.

\section{Data \& methods}

The study used the 2013 Nigeria Demographic and Health Survey (NDHS) data collected between February and July, 2013. It is a representative sample from 36 states of Nigeria and the Federal Capital Territory (FCT). The purpose of the NDHS is to provide information on population and health performance and behaviour among diverse subgroups in the country. The survey employed stratified 3-staged cluster design with sampling conducted at the state, Local Government Authority (LGA), locality and Enumeration Area (EA) levels. A sub-sample of 8319 women aged I5-49 in the north western region was extracted from the 38,945 nationally representative sample.

The north western region of Nigeria is one of the largest of the six geopolitical zones with a landmass of about one-quarter $(25.75 \%)$ of the country, and a total population of about 48.8 million (about 25.3\% of entire country's population) and the dominant religion is Islam. It is constituted by seven states, Sokoto, Kebbi, Zanfara, Katsina, Kaduna, Kano, and Jigawa states with major ethnic groups including Hausa, and Fulani.

The outcome and explanatory variables are presented in Table I according to how they were conceptualized and measured in the study.

\begin{tabular}{|c|c|c|}
\hline Variables & Description & Categories/Codes \\
\hline \multicolumn{3}{|l|}{ Outcome variable: } \\
\hline $\begin{array}{l}\text { Current contraceptive } \\
\text { method }\end{array}$ & $\begin{array}{l}\text { Described as whether } \\
\text { respondent did something or } \\
\text { used any method to delay or } \\
\text { avoid getting pregnant at survey } \\
\text { period }\end{array}$ & Classified as not using $=1$, using $=2$ \\
\hline \multicolumn{3}{|c|}{ Background explanatory variables: } \\
\hline I. Age in 5-years & $\begin{array}{l}\text { Described as age of respondent } \\
\text { at last birthday. }\end{array}$ & $\begin{array}{l}\text { Responses classified as } 15-24=1,25-29= \\
2,30-34=3,35-39=4,40+=5\end{array}$ \\
\hline 2. State of residence & $\begin{array}{l}\text { The state in which respondent } \\
\text { lived at the time of survey }\end{array}$ & $\begin{array}{l}\text { Categorized as Sokoto }=1, \text { Kebbi }=2, \\
\text { Zamfara }=3, \text { Katsina }=4, \text { Kano }=6 \text {, and } \\
\text { Jigawa }=7\end{array}$ \\
\hline 3. Place of residence & $\begin{array}{l}\text { Type of place of respondent's } \\
\text { residence }\end{array}$ & Recoded as rural $=1$, urban $=2$ \\
\hline 4. Highest level of education & $\begin{array}{l}\text { The highest level of school } \\
\text { respondent attended }\end{array}$ & $\begin{array}{l}\text { Recoded as no education }=1 \text {, primary }=2 \text {, } \\
\text { secondary or higher }=3\end{array}$ \\
\hline 5. Religion & $\begin{array}{l}\text { This is the type of religion } \\
\text { professed by respondent }\end{array}$ & $\begin{array}{l}\text { Responses recoded as Islam }=1 \text {, and } \\
\text { Christianity }=2\end{array}$ \\
\hline
\end{tabular}




\begin{tabular}{|c|c|c|}
\hline 6. Wealth index & $\begin{array}{l}\text { This represents the } \\
\text { socioeconomic class of the } \\
\text { respondent derived as a } \\
\text { cumulative index from amenities } \\
\text { and possessions in the household }\end{array}$ & $\begin{array}{l}\text { This is recoded as poorest/poorer }=1 \text {, } \\
\text { middle }=2 \text {, richer }=3 \text {, and richest }=4\end{array}$ \\
\hline 7. no of co-wives & $\begin{array}{l}\text { Defined as number of other } \\
\text { wives married to husband of } \\
\text { respondent }\end{array}$ & $\begin{array}{l}\text { Dichotomized as no other wives }=1 \text {, and } \\
\text { one or more }=2\end{array}$ \\
\hline 8. Living arrangement & $\begin{array}{l}\text { Captured as whether } \\
\text { respondent was residing with } \\
\text { husband/partner at the time of } \\
\text { study }\end{array}$ & $\begin{array}{l}\text { Categorized as wife living with husband }=1 \text {, } \\
\text { wife living elsewhere }=2\end{array}$ \\
\hline 9. Number of siblings & $\begin{array}{l}\text { Defined as the total number of } \\
\text { siblings of respondent }\end{array}$ & $\begin{array}{l}\text { Categorized as none/two or less }=1 \text {, three } \\
\text { to five }=2 \text {, six or more }=3\end{array}$ \\
\hline $\begin{array}{l}\text { 10. Birth order of } \\
\text { respondent }\end{array}$ & $\begin{array}{l}\text { That is the number of siblings } \\
\text { born before respondent's birth }\end{array}$ & $\begin{array}{l}\text { Recoded as first child }=1 \text {, second or third } \\
\text { child }=2, \text { forth child or higher }=3\end{array}$ \\
\hline II. Employment status & $\begin{array}{l}\text { That is respondent's working } \\
\text { status at the time of survey }\end{array}$ & Classified as not working $=1$, working $=2$ \\
\hline 12. Type of employment & $\begin{array}{l}\text { This refers to respondent's type } \\
\text { of work that is whether formal } \\
\text { or informal }\end{array}$ & $\begin{array}{l}\text { Responses were grouped as not working }= \\
\mathrm{I}, \text { informal work }=2 \text {, and formal work }=3\end{array}$ \\
\hline $\begin{array}{l}\text { I3. Earnings from } \\
\text { employment }\end{array}$ & $\begin{array}{l}\text { Defined as the reward from } \\
\text { work whether in cash or kind }\end{array}$ & $\begin{array}{l}\text { Not working }=1 \text {, cash } / \text { kind }=2 \text {, and cash } \\
\text { only }=3\end{array}$ \\
\hline 14. Husband/partner's age & $\begin{array}{l}\text { This is the age of respondent's } \\
\text { husband/partner at the time of } \\
\text { survey }\end{array}$ & $\begin{array}{l}\text { Regrouped as } 34 \text { or younger }=1,35-49= \\
2, \text { and } 50 \text { or older }=3\end{array}$ \\
\hline $\begin{array}{l}\text { I5. Husband/partner's } \\
\text { education }\end{array}$ & $\begin{array}{l}\text { Defined as husband/partner's } \\
\text { highest level of education } \\
\text { attained }\end{array}$ & $\begin{array}{l}\text { Categorized as no education/don't know }= \\
\mathrm{I} \text {, primary }=2 \text {, secondary }=3 \text {, and higher } \\
=4\end{array}$ \\
\hline $\begin{array}{l}\text { 16. Husband/partner's type } \\
\text { of work }\end{array}$ & $\begin{array}{l}\text { Defined as the type of work that } \\
\text { husband/partner was doing at } \\
\text { time of survey }\end{array}$ & $\begin{array}{l}\text { Categorized as skilled/unskilled manual }=1 \\
\text { agricultural }=2 \text {, and technical services }=3\end{array}$ \\
\hline \multicolumn{3}{|c|}{ Agents of modernity explanatory variables: } \\
\hline $\begin{array}{l}\text { 17. Household have } \\
\text { electricity }\end{array}$ & $\begin{array}{l}\text { Respondent's household have } \\
\text { electricity }\end{array}$ & $\begin{array}{l}\text { Responses dichotomized as no }=1 \text {, and yes } \\
=2\end{array}$ \\
\hline 18. Household have radio & $\begin{array}{l}\text { Respondent's household have } \\
\text { radio }\end{array}$ & $\begin{array}{l}\text { Responses dichotomized as no }=\mathrm{I} \text {, and yes } \\
=2\end{array}$ \\
\hline 19. Household have TV & $\begin{array}{l}\text { Respondent's household have } \\
\text { TV }\end{array}$ & $\begin{array}{l}\text { Responses dichotomized as no }=I \text {, and yes } \\
=2\end{array}$ \\
\hline $\begin{array}{l}\text { 20. Household have cable } \\
\text { TV }\end{array}$ & $\begin{array}{l}\text { Respondent's household have } \\
\text { cable TV }\end{array}$ & $\begin{array}{l}\text { Responses dichotomized as no }=1 \text {, and yes } \\
=2\end{array}$ \\
\hline 21. Has mobile phone & Respondent has mobile phone & $\begin{array}{l}\text { Responses dichotomized as no }=1 \text {, and yes } \\
=2\end{array}$ \\
\hline $\begin{array}{l}\text { 22. Multiple exposure to } \\
\text { agents of modernity }\end{array}$ & $\begin{array}{l}\text { Respondent's household having } \\
\text { more than one agent of } \\
\text { modernity items }\end{array}$ & $\begin{array}{l}\text { Regrouped as none }=1 \text {, one }=2, \text { two }=3 \text {, } \\
\text { more than two }=4\end{array}$ \\
\hline \multicolumn{3}{|c|}{ Frequency of exposure to agents of modernity: } \\
\hline $\begin{array}{l}23 \text {. Frequency listened to } \\
\text { radio }\end{array}$ & $\begin{array}{l}\text { Frequency that respondent } \\
\text { listened to radio }\end{array}$ & $\begin{array}{l}\text { Responses recoded as not at all }=I \text {, less } \\
\text { than once a week }=2 \text {, at least once a week } \\
=3 \text {, and almost every day }=4\end{array}$ \\
\hline
\end{tabular}




\begin{tabular}{|c|c|c|}
\hline 24. Frequency watched TV & $\begin{array}{l}\text { Frequency that respondent } \\
\text { watched TV }\end{array}$ & $\begin{array}{l}\text { Responses recoded as not at all }=1 \text {, less } \\
\text { than once a week }=2 \text {, at least once a week } \\
=3 \text {, and almost every day }=4 \text {. }\end{array}$ \\
\hline \multicolumn{3}{|l|}{ Fertility Behavior: } \\
\hline $\begin{array}{l}\text { 25. Number of living } \\
\text { children }\end{array}$ & $\begin{array}{l}\text { That is respondent's number of } \\
\text { living children at the time of } \\
\text { survey }\end{array}$ & $\begin{array}{l}\text { Regrouped as three or more }=1 \text {, and two } \\
\text { or one }=2\end{array}$ \\
\hline $\begin{array}{l}\text { 26. Preference for another } \\
\text { child }\end{array}$ & $\begin{array}{l}\text { Respondent favor having another } \\
\text { child }\end{array}$ & $\begin{array}{l}\text { Responses dichotomized as favorable to } \\
\text { another child }=1 \text {, and not favorable to } \\
\text { another child }=2\end{array}$ \\
\hline
\end{tabular}

\section{Methods of analysis}

Data analysis was done at the univariate, bivariate, and multivariate levels. In order to provide more insight on the effects of explanatory factors on contraceptive use, analysis controlled for residence (rural vs. urban). The unit of analysis was women aged 15-49 married or living with partner at the time of survey. Univariate analysis produced weighted and unweighted descriptive statistics across all variables used in this study. Weight was not applied in bivariate (correlation), and multivariate (regression) analyses because it is inappropriate for estimating correlation and regression coefficients (Rutstein and Rojas, 2006). Bivariate analyses show significant association between contraceptive use and explanatory factors disaggregated by rural and urban respondents.

Due to the dichotomous nature of the outcome variable, contraceptive use, logistics regression analytical technique was employed to show its relationships with the predictors. Before embarking on multivariate analysis, multicollinearity diagnostics
(Appendix I) was conducted to ensure that there were no multicollinearity issues among the variables. Critical values for high collinearity include when; pairwise correlation factor exceeds 0.7 , condition index $(\mathrm{Cl})$ has eigenvalues greater than 30 , and variance inflation factor (VIF) is greater than 10 (Dormann, et al., 20I2). Two variables that were dropped from multivariate analysis after collinearity diagnosis test, were type of earnings from employment, and cumulative index of agents of modernity. Multivariate analysis used block modeling to establish the relationships between contraceptive use and explanatory factors. In order to establish direct and indirect effects, Model I examined only the relationships between the outcome variable contraceptive use and socioeconomic predictors, model II examined the relationships between contraceptive use and agents of modernity, Model III teased out only the relationships between fertility behaviors and the outcome variable, and Model IV is the full model that includes all predictors.

Results

Descriptive statistics

Table 2: Frequency distribution of married women and those in union in north-west region of Nigeria by predictors

\begin{tabular}{|c|c|c|c|c|}
\hline \multirow[b]{2}{*}{ Variables } & \multicolumn{2}{|c|}{ Unweighted } & \multicolumn{2}{|c|}{ Weighted } \\
\hline & Frequency & $\begin{array}{l}\text { Valid Percent } \\
\text { (\%) }\end{array}$ & Frequency & $\begin{array}{l}\text { Valid Percent } \\
\text { (\%) }\end{array}$ \\
\hline \multicolumn{5}{|c|}{ MARRIED WOMEN BACKGROUND VARIABLES } \\
\hline \multicolumn{5}{|c|}{ Women age in groups } \\
\hline 24 or less & 2507 & 30.1 & 3020 & 30.1 \\
\hline $25-29$ & 1685 & 20.3 & 2027 & 20.2 \\
\hline $30-34$ & 1274 & 15.3 & $|54|$ & 15.4 \\
\hline $35-39$ & 1124 & 13.5 & 1358 & 13.5 \\
\hline $40+$ & 1729 & 20.8 & 2089 & 20.8 \\
\hline \multicolumn{5}{|l|}{ State of residence } \\
\hline Sokoto & $|1| 3 \mid$ & 13.6 & 956 & 9.5 \\
\hline Kebbi & 1041 & 12.5 & 1074 & 10.7 \\
\hline Zamfara & 1089 & 13.1 & 1226 & 12.2 \\
\hline Katsina & 1191 & 14.3 & 1408 & 14.0 \\
\hline Kaduna & 944 & 11.3 & 1594 & 15.9 \\
\hline Kano & 1798 & 21.6 & 2521 & 25.1 \\
\hline Jigawa & 1125 & 13.5 & 1256 & 12.5 \\
\hline
\end{tabular}


Place of residence

\begin{tabular}{|c|c|c|c|c|}
\hline Rural & 6637 & 79.8 & 7699 & 76.7 \\
\hline Urban & 1682 & 20.2 & 2336 & 23.3 \\
\hline \multicolumn{5}{|l|}{ Highest educational level } \\
\hline No education & 6580 & 79.1 & 7683 & 76.6 \\
\hline Primary & 912 & 11.0 & 1156 & 11.5 \\
\hline Secondary or higher & 827 & 9.9 & 1195 & 11.9 \\
\hline \multicolumn{5}{|l|}{ Religion of respondent } \\
\hline Islam & 7813 & 95.1 & 9199 & 92.7 \\
\hline Christianity & 401 & 4.9 & 719 & 7.3 \\
\hline \multicolumn{5}{|l|}{ Wealth index } \\
\hline Poorest- poorer & 59.2 & 70.9 & 6894 & 68.7 \\
\hline Middle & $|20|$ & 14.4 & 1472 & 14.7 \\
\hline Richer & 779 & 9.4 & 1027 & 10.2 \\
\hline Richest & 437 & 5.3 & 642 & 6.4 \\
\hline \multicolumn{5}{|l|}{ No of co-wives } \\
\hline No other wives & 4578 & 55.2 & 5617 & 56.2 \\
\hline One of more & 3708 & 44.8 & 4380 & 43.8 \\
\hline \multicolumn{5}{|l|}{ Living arrangement } \\
\hline \multirow{2}{*}{$\begin{array}{l}\text { Wife living with husband } \\
\text { Wife living elsewhere }\end{array}$} & 7867 & 95.3 & 9531 & 95.6 \\
\hline & 391 & 4.7 & 435 & 4.4 \\
\hline \multicolumn{5}{|l|}{ Number of siblings of respondents } \\
\hline None-two or less & 1414 & 17.0 & 1749 & 17.4 \\
\hline Three to five & 3010 & 36.2 & 3778 & 37.7 \\
\hline Six or more & 3891 & 46.8 & 4502 & 44.9 \\
\hline \multicolumn{5}{|l|}{ Birth order of respondent } \\
\hline First child & 1854 & 23.2 & 2236 & 23.2 \\
\hline Second or third child & 3140 & 39.2 & 3856 & 40.0 \\
\hline Forth child or higher & 3010 & 37.6 & 3550 & 36.8 \\
\hline \multicolumn{5}{|l|}{ Employment status } \\
\hline No working & $317 \mid$ & 38.3 & 3778 & 37.8 \\
\hline Working & 5106 & 61.7 & 6206 & 62.2 \\
\hline \multicolumn{5}{|l|}{ Type of employment } \\
\hline Not working- others Skilled-unskilled & 3022 & 36.4 & 3617 & 36.1 \\
\hline manual & 1183 & 14.3 & 1404 & 14.0 \\
\hline \multicolumn{5}{|l|}{ Agricultural } \\
\hline \multirow[t]{2}{*}{ Technical-services } & 144 & 1.7 & 185 & 1.8 \\
\hline & 3951 & 47.6 & 4807 & 48.0 \\
\hline \multicolumn{5}{|l|}{ Earnings from employment } \\
\hline Not working & 3034 & 36.5 & 3636 & 36.2 \\
\hline Cash or kind & 393 & 4.7 & 466 & 4.6 \\
\hline Cash only & 4892 & 58.8 & 5933 & 59.1 \\
\hline \multicolumn{5}{|l|}{ Husband/partner age in groups } \\
\hline 34 or younger & 2091 & 25.1 & 2544 & 25.4 \\
\hline $35-49$ & 3885 & 46.7 & 4740 & 47.2 \\
\hline 50 or older & 2343 & 28.2 & 2751 & 27.4 \\
\hline \multicolumn{5}{|c|}{ Husband/partner highest educational level } \\
\hline \multicolumn{5}{|l|}{ Primary } \\
\hline Secondary & 1140 & 13.7 & 1379 & 13.8 \\
\hline \multirow{2}{*}{ Higher } & 1073 & 12.9 & |449 & 14.5 \\
\hline & 571 & 6.9 & 709 & 7.1 \\
\hline
\end{tabular}

Husband/partner type of work 
African Population Studies Vol 33, No. 2, 2019

\begin{tabular}{|c|c|c|c|c|}
\hline $\begin{array}{l}\text { Skilled-unskilled manual } \\
\text { Agricultural } \\
\text { Technical services }\end{array}$ & $\begin{array}{l}3153 \\
3394\end{array}$ & $\begin{array}{l}38.4 \\
41.4\end{array}$ & $\begin{array}{l}3780 \\
4 \mid 43\end{array}$ & $\begin{array}{l}38.2 \\
41.8\end{array}$ \\
\hline \multicolumn{5}{|l|}{ AGENTS OF MODERNITY } \\
\hline \multicolumn{5}{|l|}{ Household has electricity } \\
\hline $\begin{array}{l}\text { No } \\
\text { Yes }\end{array}$ & $\begin{array}{l}5140 \\
3168\end{array}$ & $\begin{array}{l}61.9 \\
38.1\end{array}$ & $\begin{array}{l}6124 \\
3901\end{array}$ & $\begin{array}{l}61.1 \\
38.9\end{array}$ \\
\hline \multicolumn{5}{|l|}{ Household has radio } \\
\hline $\begin{array}{l}\text { No } \\
\text { Yes }\end{array}$ & $\begin{array}{l}2389 \\
5913\end{array}$ & $\begin{array}{l}28.8 \\
71.2\end{array}$ & $\begin{array}{l}2773 \\
7243\end{array}$ & $\begin{array}{l}27.7 \\
72.3\end{array}$ \\
\hline \multicolumn{5}{|l|}{ Household has TV } \\
\hline $\begin{array}{l}\text { No } \\
\text { Yes }\end{array}$ & $\begin{array}{l}6340 \\
1955\end{array}$ & $\begin{array}{l}76.4 \\
23.6\end{array}$ & $\begin{array}{l}7488 \\
2522\end{array}$ & $\begin{array}{l}74.8 \\
25.2\end{array}$ \\
\hline \multicolumn{5}{|l|}{ Household has cable TV } \\
\hline $\begin{array}{l}\text { No } \\
\text { Yes }\end{array}$ & $\begin{array}{l}7802 \\
494\end{array}$ & $\begin{array}{l}94.0 \\
6.0\end{array}$ & $\begin{array}{l}9376 \\
632\end{array}$ & $\begin{array}{l}93.7 \\
6.3\end{array}$ \\
\hline \multicolumn{5}{|l|}{ Household has Telephone } \\
\hline $\begin{array}{l}\text { No } \\
\text { Yes }\end{array}$ & $\begin{array}{l}7872 \\
43\end{array}$ & $\begin{array}{l}95.1 \\
4.9\end{array}$ & $\begin{array}{l}9448 \\
535\end{array}$ & $\begin{array}{l}94.6 \\
5.4\end{array}$ \\
\hline \multicolumn{5}{|l|}{ Has mobile telephone } \\
\hline $\begin{array}{l}\text { No } \\
\text { Yes }\end{array}$ & $\begin{array}{l}2879 \\
5411\end{array}$ & $\begin{array}{l}34.7 \\
65.3\end{array}$ & $\begin{array}{l}3411 \\
6590\end{array}$ & $\begin{array}{l}34.1 \\
65.9 \\
\end{array}$ \\
\hline \multicolumn{5}{|c|}{ Multiple exposure to agents of modernity } \\
\hline $\begin{array}{l}\text { None } \\
\text { One } \\
\text { Two } \\
\text { More than two }\end{array}$ & $\begin{array}{l}998 \\
2120 \\
2499 \\
2702 \\
\end{array}$ & $\begin{array}{l}12.0 \\
25.5 \\
30.0 \\
32.5\end{array}$ & $\begin{array}{l}1148 \\
2488 \\
3044 \\
3355\end{array}$ & $\begin{array}{l}11.4 \\
24.8 \\
30.3 \\
33.4 \\
\end{array}$ \\
\hline \multicolumn{5}{|c|}{ FREQUENCY OF EXPOSURE TO MODERNITY } \\
\hline \multicolumn{5}{|l|}{ Frequency listened to radio } \\
\hline $\begin{array}{l}\text { Not at all } \\
\text { Less than once a week } \\
\text { At least once a week }\end{array}$ & $\begin{array}{l}3570 \\
2182\end{array}$ & $\begin{array}{l}43.1 \\
26.4\end{array}$ & $\begin{array}{l}4029 \\
2819\end{array}$ & $\begin{array}{l}40.3 \\
28.2 \\
31.5\end{array}$ \\
\hline \multicolumn{5}{|l|}{ Frequency watch TV } \\
\hline $\begin{array}{l}\text { Not at all } \\
\text { Less than once a week } \\
\text { At least once a week }\end{array}$ & $\begin{array}{l}6305 \\
965 \\
1007 \\
\end{array}$ & $\begin{array}{l}76.2 \\
11.7\end{array}$ & $\begin{array}{r}7303 \\
1355 \\
1332 \\
\end{array}$ & $\begin{array}{l}73.1 \\
13.6 \\
13.3 \\
\end{array}$ \\
\hline \multicolumn{5}{|l|}{ FERTILITY BEHAVIOR } \\
\hline $\begin{array}{l}\text { Three or more } \\
\text { Two or less }\end{array}$ & $\begin{array}{l}4967 \\
3352\end{array}$ & $\begin{array}{l}59.7 \\
40.3\end{array}$ & $\begin{array}{l}5974 \\
4060\end{array}$ & $\begin{array}{l}59.5 \\
40.5\end{array}$ \\
\hline \multicolumn{5}{|l|}{ Fertility preference } \\
\hline $\begin{array}{l}\text { Favorable to another child } \\
\text { Not favorable to another child }\end{array}$ & $\begin{array}{l}7257 \\
1020\end{array}$ & $\begin{array}{l}87.2 \\
12.3\end{array}$ & $\begin{array}{l}8744 \\
1243\end{array}$ & $\begin{array}{l}87.6 \\
12.4\end{array}$ \\
\hline \multicolumn{5}{|l|}{ Contraceptive status } \\
\hline $\begin{array}{l}\text { Not using } \\
\text { Using }\end{array}$ & $\begin{array}{l}8049 \\
270\end{array}$ & $\begin{array}{l}96.8 \\
3.2\end{array}$ & $\begin{array}{l}9607 \\
427\end{array}$ & $\begin{array}{l}95.7 \\
4.3\end{array}$ \\
\hline
\end{tabular}

Weighted univariate results in Table 2 show that the majority of married women were aged 34 or younger (65.7\%), and were fairly distributed across the states, with the majority from Kano (25.1\%), followed by http://aps.journals.ac.za
Kaduna (I5.9\%), Katsina (I4.0\%), Jigawa (I2.5\%), Zamfara (I $2.2 \%)$, Kebbi (I0.7\%) and Sokoto (9.5\%). Most respondents were of rural residence (76.7\%), had no education (76.6\%), were of Islamic religion 
$(92.7 \%)$, and were of poorest/poorer wealth status (68.7\%). Less than half (43.8\%) had one or more cowives, the majority $(95.6 \%)$ lived with their husband/partner, and close to half (44.9\%) had 6 or more siblings. Results showed that the majority of respondent $(76.8 \%)$ were of second and lower birth order, employed (62.2\%), were involved in agriculture/technical/services work (49.8\%), and earned cash for work (59.1\%). The majority of respondents' husbands/partners were aged 49 or younger $(72.6 \%)$, uneducated/don't know (64.5\%), and significant proportion were in technical/services work (4I.8\%), followed by agricultural work (38.2\%), and skilled/unskilled manual labour (20\%).

On agents of modernity, only about a third of the respondents $(38.9 \%)$ had electricity, the majority had radio $(72.3 \%)$ and mobile phone $(65.9 \%)$, while small proportions had TV (25.2\%), cable TV (6.3\%), and house telephone $(5.4 \%)$. This study measured cumulative index of modernity i.e. the number of agents of modernity reported by respondents. The majority of respondents $(63.7 \%)$ had at least two or more agents of modernity in their household, and above half $(59.7 \%)$ reported listening to radio less than once a week or at least once a week, and

$26.9 \%$ watched TV less than once a week or at least once a week. Findings showed that the majority of respondents had three or more children $(59.5 \%)$, were favorable to having another child $(87.6 \%)$, and only a small proportion (3.2\%) used modern contraceptives.

Bivariate results

Table 3: Showing bivariate association between modern contraceptive use among married women and those in union in Nigeria by predictors

\begin{tabular}{|c|c|c|c|c|c|c|}
\hline \multirow[b]{2}{*}{ Variables } & \multicolumn{3}{|c|}{ Rural } & \multicolumn{3}{|c|}{ Urban } \\
\hline & $\begin{array}{l}\text { Not using } \\
\%\end{array}$ & Using \% & $P-$ Value & $\begin{array}{l}\text { Not using } \\
\%\end{array}$ & Using \% & $P-$ Value \\
\hline \multicolumn{7}{|c|}{ MARRIED WOMEN BACKGROUND VARIABLE } \\
\hline \multicolumn{7}{|c|}{ Women age in groups } \\
\hline $15-24$ & 99.1 & 0.9 & & 95.2 & 4.8 & \\
\hline $25-29$ & 97.9 & 2.1 & & 91.1 & 8.9 & \\
\hline $30-34$ & 98.3 & 1.7 & & 85.9 & |4.| & \\
\hline $35-39$ & 96.5 & 3.5 & & 88.2 & 11.8 & \\
\hline $40+$ & 98.3 & 1.7 & .000 & 92.1 & 7.9 & .000 \\
\hline \multicolumn{7}{|l|}{ State of residence } \\
\hline Sokoto & 99.6 & 0.4 & & 94.6 & 5.4 & \\
\hline Kebbi & 99.1 & 0.9 & & 95.5 & 4.5 & \\
\hline Zamfara & 98.3 & 1.7 & & 87.7 & 12.3 & \\
\hline Katsina & 99.5 & 0.5 & & 95.0 & 5.0 & \\
\hline Kaduna & 87.3 & 12.7 & & 73.6 & 26.4 & \\
\hline Kano & 99.8 & 0.2 & & 98.5 & 1.5 & \\
\hline Jigawa & 99.2 & 0.8 & .000 & 98.8 & 1.2 & .000 \\
\hline \multicolumn{7}{|c|}{ Highest educational level } \\
\hline No education & 99.1 & 0.9 & & 97.1 & 2.9 & \\
\hline Primary & 94.8 & 5.2 & & 90.1 & 9.9 & \\
\hline Secondary or higher & 88.9 & II.I & .000 & 80.7 & 19.3 & .000 \\
\hline \multicolumn{7}{|c|}{ Religion of respondent } \\
\hline Islam & 99.1 & 0.9 & & 95.5 & 4.5 & \\
\hline Christianity & 70.1 & 29.9 & .000 & 58.3 & 41.7 & .000 \\
\hline \multicolumn{7}{|l|}{ Wealth index } \\
\hline \multicolumn{7}{|l|}{ Middle } \\
\hline Richer & 95.4 & 4.6 & & 97.5 & 2.5 & \\
\hline \multirow[t]{2}{*}{ Richest } & 91.4 & 8.6 & & 91.1 & 8.9 & \\
\hline & 93.5 & 6.5 & .000 & 78.3 & 21.7 & .000 \\
\hline \multicolumn{7}{|l|}{ No of co-wives } \\
\hline No other wives & 97.7 & 2.3 & & 88.7 & 11.3 & \\
\hline One or more & 98.9 & I.I & .000 & 95.1 & 4.9 & .000 \\
\hline
\end{tabular}


African Population Studies Vol 33, No. 2, 2019

\begin{tabular}{|c|c|c|c|c|c|c|}
\hline $\begin{array}{l}\text { Wife living with husband } \\
\text { Wife living elsewhere }\end{array}$ & 98.5 & 1.8 & .728 & 93.8 & 6.2 & .412 \\
\hline \multicolumn{7}{|c|}{ Number of siblings of respondent } \\
\hline \multicolumn{6}{|l|}{ Three to five } & \\
\hline Six or more & $\begin{array}{l}97.9 \\
98.4\end{array}$ & $\begin{array}{l}2.1 \\
1.6\end{array}$ & .270 & $\begin{array}{l}87.8 \\
93.6\end{array}$ & $\begin{array}{l}12.2 \\
6.4 \\
\end{array}$ & .000 \\
\hline \multicolumn{7}{|c|}{ Birth order of respondent } \\
\hline First child & 98.2 & 1.8 & & 88.0 & 12.0 & \\
\hline \multirow{2}{*}{$\begin{array}{l}\text { Second or third child } \\
\text { Forth child or higher }\end{array}$} & 98.2 & 1.8 & & 91.1 & 9.0 & \\
\hline & 98.3 & 1.7 & .901 & 92.1 & 7.9 & .103 \\
\hline \multicolumn{7}{|l|}{ Employment status } \\
\hline $\begin{array}{l}\text { No working } \\
\text { Working }\end{array}$ & $\begin{array}{l}98.6 \\
98.0\end{array}$ & $\begin{array}{l}1.4 \\
2.0\end{array}$ & .069 & $\begin{array}{l}96.1 \\
88.3\end{array}$ & $\begin{array}{l}3.9 \\
11.7\end{array}$ & .000 \\
\hline \multicolumn{7}{|l|}{ Type of employment } \\
\hline \multicolumn{7}{|l|}{ Skilled-unskilled manual } \\
\hline \multirow[t]{2}{*}{$\begin{array}{l}\text { Agricultural } \\
\text { Technical-services }\end{array}$} & 99.4 & 0.6 & & 96.2 & 3.8 & \\
\hline & $\begin{array}{l}95.7 \\
97.7\end{array}$ & $\begin{array}{l}4.3 \\
2.3 \\
\end{array}$ & .000 & $\begin{array}{l}100.0 \\
86.6\end{array}$ & 13.4 & .000 \\
\hline \multicolumn{7}{|c|}{ Earnings from employment } \\
\hline Not working & 98.6 & 1.4 & & 96.0 & 4.0 & \\
\hline Cash or kind & 99.7 & 0.3 & & 90.7 & 9.3 & \\
\hline Cash only & 97.9 & 2.1 & .013 & 88.5 & 11.5 & .000 \\
\hline \multicolumn{7}{|c|}{ Husband/partner age in groups } \\
\hline 34 or younger & 98.5 & 1.5 & & 92.6 & 7.4 & \\
\hline $35-49$ & 97.9 & 2.1 & & 88.4 & 11.6 & \\
\hline 50 or older & 98.6 & 1.4 & .106 & 93.7 & 6.3 & .002 \\
\hline \multicolumn{7}{|c|}{ Husband/partner highest educational level } \\
\hline \multicolumn{6}{|l|}{ Primary } & \\
\hline Secondary & 96.9 & 3.1 & & 92.6 & 7.4 & \\
\hline \multirow[t]{2}{*}{ Higher } & 95.3 & 4.7 & & 87.4 & 12.6 & \\
\hline & 92.3 & 7.7 & .000 & 80.9 & 19.1 & .000 \\
\hline \multicolumn{7}{|c|}{ Husband/partner type of work } \\
\hline \multirow[t]{2}{*}{$\begin{array}{l}\text { Skilled-unskilled manual } \\
\text { Agricultural } \\
\text { Technical services }\end{array}$} & 98.6 & 1.4 & & 92.4 & 7.6 & \\
\hline & 98.2 & 1.8 & & 96.3 & 3.7 & 100 \\
\hline \multicolumn{7}{|c|}{ AGENTS OF MODERNITY } \\
\hline \multicolumn{7}{|l|}{ Household has electricity } \\
\hline No & 98.3 & 1.7 & & 98.1 & 1.9 & \\
\hline Yes & 98.1 & 1.9 & .618 & 89.6 & 10.4 & .000 \\
\hline \multicolumn{7}{|l|}{ Household has radio } \\
\hline No & 98.5 & 1.5 & & 97.7 & 2.3 & \\
\hline Yes & 98.1 & 1.9 & .315 & 89.9 & 10.1 & .000 \\
\hline \multicolumn{7}{|l|}{ Household has TV } \\
\hline No & 98.5 & 1.5 & & 98.6 & 1.4 & \\
\hline Yes & 96.5 & 3.5 & .000 & 87.1 & 12.9 & .000 \\
\hline
\end{tabular}




\begin{tabular}{|c|c|c|c|c|c|c|}
\hline No & 98.3 & 1.7 & & 92.6 & 7.4 & \\
\hline Yes & 95.9 & 4.1 & .033 & 84.4 & 15.6 & .000 \\
\hline \multicolumn{7}{|c|}{ Household has Telephone } \\
\hline No & 98.2 & 1.8 & & 90.6 & 9.4 & \\
\hline Yes & 99.2 & 0.8 & .252 & 97.5 & 2.5 & .003 \\
\hline \multicolumn{7}{|l|}{ Has mobile telephone } \\
\hline No & 98.2 & 1.8 & & 99.6 & 0.4 & \\
\hline Yes & 98.2 & 1.8 & .978 & 89.2 & 10.8 & .000 \\
\hline \multicolumn{7}{|c|}{ Multiple exposure to agents of modernity } \\
\hline None & 98.2 & 1.8 & & 100.0 & 1.8 & \\
\hline One & 98.3 & 1.7 & & 98.2 & 0.5 & \\
\hline Two & 98.6 & 1.4 & & 99.5 & 11.2 & \\
\hline More than two & 97.5 & 2.5 & .107 & 88.8 & & .000 \\
\hline \multicolumn{7}{|c|}{ FREQUENCY OF EXPOSURE TO MODERNITY } \\
\hline \multicolumn{7}{|c|}{ Frequency listened to radio } \\
\hline Not at all & 98.9 & 1.1 & & 96.7 & 3.3 & \\
\hline Less than once a week & 97.6 & 2.4 & & 85.7 & 14.3 & \\
\hline & \multicolumn{3}{|c|}{ At least once a week } & 91.3 & & .000 \\
\hline \multicolumn{7}{|l|}{ Frequency watch TV } \\
\hline Not at all & 98.7 & 1.3 & & 97.8 & 2.2 & \\
\hline \multirow{2}{*}{$\begin{array}{l}\text { Less than once a week } \\
\text { At least once a week }\end{array}$} & 95.7 & 4.3 & & 84.7 & 15.3 & \\
\hline & 95.2 & 4.8 & .000 & 88.1 & 11.9 & .000 \\
\hline \multicolumn{7}{|c|}{ Preference for another child } \\
\hline \multirow{3}{*}{$\begin{array}{l}\text { Favorable to another child } \\
\text { Not favorable to another } \\
\text { child }\end{array}$} & 98.6 & 1.4 & & 92.7 & 7.3 & \\
\hline & 96.0 & & & & & \\
\hline & & 4.0 & .000 & 82.7 & 17.3 & .000 \\
\hline \multicolumn{7}{|l|}{ No of living children } \\
\hline \multirow{2}{*}{$\begin{array}{l}\text { Three or more } \\
\text { Two or less }\end{array}$} & 97.7 & 2.3 & & 89.6 & 10.4 & \\
\hline & 99.0 & 1.0 & .000 & 93.2 & 6.8 & .012 \\
\hline
\end{tabular}

Findings on bivariate analysis presented in Table 3 showed that most predictors were significantly associated with contraceptive use with consistently more proportion of users in urban than in rural areas. Also, more predictors of contraceptive use were significant for urban than rural respondents. Background factors significantly associated (at minimum .05 level) with contraceptive use both in urban and rural areas were respondent's age, state of residence, level of education, religion, wealth index, and number of co-wives. Other background factors significantly associated with contraceptive use at both urban and rural areas were type of employment, earnings from employment, and husbands' highest education. Background factors that were significantly associated with contraceptive use for urban residents only were number of siblings of respondents, employment status, husband/partner's age, and husband's type of work.

The agents of modernity significantly associated (at minimum .05 level) with contraceptive use for both urban and rural residents were household had TV, and cable TV, and frequency listened to radio, and TV. While the following predictors household has; electricity, radio, telephone, mobile telephone, and multiple exposure to agents of modernity were significantly associated with modern contraceptive use in urban areas only. Results showed that fertility preference and number of living children were significantly associated with modern contraceptive use in both urban and rural areas. 
Multivariate results

Key Determinants of Modern Contraceptive Use in Rural Areas

Table 4: The Odds that married women and those in union aged $15-49$ living in rural areas used modern contraceptives by predictors

\begin{tabular}{|c|c|c|}
\hline & Model I & Model 2 \\
\hline Variables & OR $(95 \% \mathrm{Cl})$ & OR $(95 \% \mathrm{Cl})$ \\
\hline \multicolumn{3}{|c|}{ MARRIED WOMEN BACKGROUND VARIABLES } \\
\hline \multicolumn{3}{|c|}{ Age of respondent } \\
\hline (single years) & $1.11(.914,1.36)$ & -- \\
\hline \multicolumn{3}{|l|}{ State of residence } \\
\hline Sokoto & 1.00 & -- \\
\hline Kebbi & $3.03(.783,11.76)$ & -- \\
\hline Zamfara & $6.47(1.83,22.96)$ *** & -- \\
\hline Katsina & $\mathrm{I} .30(.296,5.7 \mathrm{I})$ & -- \\
\hline Kaduna & $12.14(3.45,42.80) * * *$ & -- \\
\hline Kano & $.543(.107,2.75)$ & -- \\
\hline Jigawa & $2.54(.662,9.73)$ & -- \\
\hline \multicolumn{3}{|l|}{ Highest educational level } \\
\hline No education & 1.00 & -- \\
\hline Primary & $2.26(I .2 I, 4.2 I) *$ & -- \\
\hline Secondary or higher & $1.35(.611,2.98)$ & -- \\
\hline \multicolumn{3}{|l|}{ Religion of respondent } \\
\hline Islam & 1.00 & -- \\
\hline Christianity & $9.22(4.58,18.56) * * * *$ & -- \\
\hline \multicolumn{3}{|l|}{ Wealth index } \\
\hline Poorest- poorer & 1.00 & -- \\
\hline Middle & $1.26(.704,2.25)$ & -- \\
\hline Richer & $3.13(1.31,7.49) *$ & -- \\
\hline Richest & $1.92(.305,12.14)$ & -- \\
\hline \multicolumn{3}{|l|}{ No of co-wives } \\
\hline No other wives & 1.00 & -- \\
\hline One or more & $.760(.458,1.26)$ & -- \\
\hline \multicolumn{3}{|l|}{ Living arrangement } \\
\hline \multirow{2}{*}{$\begin{array}{l}\text { Wife living with husband } \\
\text { Wife living elsewhere }\end{array}$} & 1.00 & -- \\
\hline & $\mathrm{I} .42(.501,4.02)$ & -- \\
\hline \multicolumn{3}{|c|}{ Number of siblings of respondent } \\
\hline None-two or less & 1.00 & -- \\
\hline Three to five & $2.23(1.06,4.67) *$ & -- \\
\hline Six or more & $2.5 \mathrm{I}(1.16,5.45) *$ & -- \\
\hline \multicolumn{3}{|c|}{ Birth order of respondent } \\
\hline First child & 1.00 & -- \\
\hline \multirow{2}{*}{$\begin{array}{l}\text { Second or third child } \\
\text { Forth child or higher }\end{array}$} & $.777(.448,1.35)$ & -- \\
\hline & $.646(.358,1.16)$ & -- \\
\hline \multicolumn{3}{|l|}{ Employment status } \\
\hline No working & 1.00 & -- \\
\hline Working & $.634(.146,2.76)$ & -- \\
\hline \multicolumn{3}{|l|}{ Type of employment } \\
\hline Not working- others & 1.00 & -- \\
\hline \multirow{4}{*}{$\begin{array}{l}\text { Skilled-unskilled manual } \\
\text { Agricultural } \\
\text { Technical-services }\end{array}$} & & \\
\hline & $.837(.006,123.21)$ & -- \\
\hline & & \\
\hline & $\begin{array}{l}.492(.003,75.92) \\
1.39(.010,192.26)\end{array}$ & $\begin{array}{l}-- \\
--\end{array}$ \\
\hline
\end{tabular}




\begin{tabular}{|c|c|c|}
\hline \multicolumn{3}{|l|}{ Earnings from employment } \\
\hline Not working & 1.00 & -- \\
\hline Cash or kind & $.299(.001,60.00)$ & -- \\
\hline Cash only & I. $.78(.013,242.85)$ & -- \\
\hline \multicolumn{3}{|l|}{ Husband Age } \\
\hline (single years) & $1.00(.973,1.04)$ & -- \\
\hline \multicolumn{3}{|c|}{ Husband highest educational level } \\
\hline $\begin{array}{l}\text { No education-don't know } \\
\text { Primary }\end{array}$ & 1.00 & -- \\
\hline Secondary & $\mathrm{I} .88(\mathrm{I} .0 \mathrm{I}, 3.5 \mathrm{I})^{*}$ & -- \\
\hline Higher & \multirow{2}{*}{$\begin{array}{l}\mathrm{I} .00(.482,2.09) \\
\mathrm{I} .75(.694,4.44)\end{array}$} & -- \\
\hline & & -- \\
\hline \multicolumn{3}{|c|}{ Respondents husband type of work } \\
\hline $\begin{array}{l}\text { Skilled-unskilled manual } \\
\text { Agricultural }\end{array}$ & 1.00 & -- \\
\hline Technical services & $\begin{array}{l}.948(.488,1.84) \\
1.49(.765,2.89)\end{array}$ & $\begin{array}{l}-- \\
--\end{array}$ \\
\hline \multicolumn{3}{|l|}{ AGENTS OF MODERNITY } \\
\hline \multicolumn{3}{|l|}{ Household has electricity } \\
\hline No & -- & 1.00 \\
\hline Yes & -- & $.519(.211,1.28)$ \\
\hline \multicolumn{3}{|l|}{ Household has radio } \\
\hline No & -- & 1.00 \\
\hline & -- & $1.13(.431,2.96)$ \\
\hline \multicolumn{3}{|l|}{ Household has TV } \\
\hline No & -- & 1.00 \\
\hline Yes & -- & $1.68(.752,3.75)$ \\
\hline \multicolumn{3}{|l|}{ Household has cable TV } \\
\hline No & -- & 1.00 \\
\hline & -- & $1.14(.44 \mathrm{I}, 2.96)$ \\
\hline \multicolumn{3}{|l|}{$\begin{array}{l}\text { Yes } \\
\text { Household has Telephone }\end{array}$} \\
\hline No & -- & 1.00 \\
\hline Yes & -- & $.322(.065,1.59)$ \\
\hline \multicolumn{3}{|l|}{ Has mobile telephone } \\
\hline No & -- & 1.00 \\
\hline Yes & -- & $.850(.332,2.18)$ \\
\hline \multicolumn{3}{|c|}{ Multiple exposure to agents of modernity } \\
\hline None & -- & 1.00 \\
\hline One & -- & $.929(.322,2.68)$ \\
\hline Two & -- & $.743(.120,4.59)$ \\
\hline More than two & -- & $1.16(.082,16.27)$ \\
\hline \multicolumn{3}{|c|}{ FREQUENCY OF EXPOSURE TO MODERNITY } \\
\hline \multicolumn{3}{|c|}{ Frequency listened to radio } \\
\hline Not at all & -- & 1.00 \\
\hline Less than once a week & -- & $1.64(1.00,2.67) *$ \\
\hline At least once a week & - & $1.36(.808,2.29)$ \\
\hline Frequency watch TV & & \\
\hline Not at all & -- & 1.00 \\
\hline Less than once a week & -- & $3.06(1.81,5.19)$ **** \\
\hline At least once a week & -- & $3.37(1.80,6.28) * * *$ \\
\hline FERTILITY BEHAVIOUR & & \\
\hline Preference for another child & & \\
\hline
\end{tabular}


African Population Studies Vol 33, No. 2, 2019

Favorable to another child

Not favorable to another

child

No of living children

Three or more

Two or less

Note: Level of Significance; ${ }^{*} \mathrm{p}<.05$, ${ }^{* *} \mathrm{p}<.0 \mathrm{I},{ }^{* * *} \mathrm{p}<.00 \mathrm{I}$; Model I: Chi-square $=365.1 \mathrm{I} 3,-2$ Log likelihood $=742.235^{\mathrm{a}}$, Nagelkerke R Square $=.350$ (35\%); Model 2: Chi-square $=55.165,-2$ Log likelihood $=1093.804^{\mathrm{a}}$, Nagelkerke R Square $=.052(5.2 \%)$;

Table 4: The Odds that married women and those in union aged $15-49$ living in rural areas used modern contraceptives by predictors (continued)

\begin{tabular}{|c|c|c|}
\hline & Model 3 & Model 4 \\
\hline Variables & OR $(95 \% \mathrm{Cl})$ & OR $(95 \% \mathrm{Cl})$ \\
\hline \multicolumn{3}{|c|}{ MARRIED WOMEN BACKGROUND VARIABLES } \\
\hline \multicolumn{3}{|c|}{ Age of respondent } \\
\hline (single years) & -- & $.890(.703,1.13)$ \\
\hline \multicolumn{3}{|l|}{ State of residence } \\
\hline Sokoto & -- & 1.00 \\
\hline Kebbi & -- & $3.07(.773,12.22)$ \\
\hline Zamfara & -- & $8.95(2.44,32.82) * *$ \\
\hline Katsina & -- & $1.60(.355,7.25)$ \\
\hline Kaduna & -- & I $3.45(3.64,49.68) * * *$ \\
\hline Kano & -- & $.494(.095,2.58)$ \\
\hline Jigawa & -- & $2.99(.763,11.72)$ \\
\hline \multicolumn{3}{|l|}{ Highest educational level } \\
\hline \multicolumn{3}{|l|}{ No education } \\
\hline Primary & -- & 1.00 \\
\hline \multirow[t]{2}{*}{ Secondary or higher } & -- & $2.24(1.17,4.28) *$ \\
\hline & -- & $1.39(.597,3.27)$ \\
\hline \multicolumn{3}{|l|}{ Religion of respondent } \\
\hline Islam & -- & 1.00 \\
\hline Christianity & -- & $10.22(4.77,21.92) * * *$ \\
\hline \multicolumn{3}{|l|}{ Wealth index } \\
\hline Poorest- poorer & -- & 1.00 \\
\hline Middle & -- & $1.26(.649,2.48)$ \\
\hline Richer & -- & $6.08(1.94,18.99) * *$ \\
\hline Richest & -- & $5.7 \mathrm{I}(.688,47.4 \mathrm{I})$ \\
\hline \multicolumn{3}{|l|}{ No of co-wives } \\
\hline No other wives & -- & 1.00 \\
\hline One or more & -- & $.838(.496,1.42)$ \\
\hline \multicolumn{3}{|l|}{ Living arrangement } \\
\hline \multirow{2}{*}{$\begin{array}{l}\text { Wife living with husband } \\
\text { Wife living elsewhere }\end{array}$} & -- & 1.00 \\
\hline & -- & $1.69(.593,4.82)$ \\
\hline \multicolumn{3}{|c|}{ Number of siblings of respondent } \\
\hline None-two or less & -- & 1.00 \\
\hline Three to five & -- & $2.12(1.00,4.48) *$ \\
\hline Six or more & -- & $2.4 I(1.09,5.28) *$ \\
\hline \multicolumn{3}{|c|}{ Birth order of respondent } \\
\hline \multirow{3}{*}{$\begin{array}{l}\text { First child } \\
\text { Second or third child } \\
\text { Forth child or higher }\end{array}$} & -- & 1.00 \\
\hline & -- & $.854(.485,1.50)$ \\
\hline & -- & $.618(.334,1.14)$ \\
\hline
\end{tabular}




\begin{tabular}{|c|c|c|}
\hline \multicolumn{3}{|l|}{ Employment status } \\
\hline No working & -- & 1.00 \\
\hline Working & -- & $.64 I(.140,2.92)$ \\
\hline \multicolumn{3}{|l|}{ Type of employment } \\
\hline Not working- others & -- & 1.00 \\
\hline \multicolumn{3}{|l|}{ Skilled-unskilled manual } \\
\hline Agricultural & -- & $.897(.002,372.91)$ \\
\hline \multicolumn{3}{|l|}{ Technical-services } \\
\hline & -- & $.430(.001,188.758)$ \\
\hline & -- & $1.45(.004,573.234)$ \\
\hline \multicolumn{3}{|l|}{ Earnings from employment } \\
\hline Not working & -- & 1.00 \\
\hline Cash or kind & -- & $.320(.001,170.649)$ \\
\hline Cash only & -- & $1.92(.005,742.947)$ \\
\hline \multicolumn{3}{|l|}{ Husband Age } \\
\hline (single years) & -- & $1.01(.973,1.04)$ \\
\hline \multicolumn{3}{|c|}{ Husband highest educational level } \\
\hline No education-don't know & -- & 1.00 \\
\hline \multicolumn{3}{|l|}{ Primary } \\
\hline Secondary & -- & $1.81(.951,3.45)$ \\
\hline \multirow{2}{*}{ Higher } & -- & $1.02(.468,2.21)$ \\
\hline & -- & $1.78(.685,4.64)$ \\
\hline \multicolumn{3}{|c|}{ Respondents husband type of work } \\
\hline Skilled-unskilled manual & -- & 1.00 \\
\hline \multicolumn{3}{|l|}{ Agricultural } \\
\hline \multirow[t]{2}{*}{ Technical services } & -- & $.910(.460,1.79)$ \\
\hline & -- & $1.53(.781,3.01)$ \\
\hline \multicolumn{3}{|l|}{ AGENTS OF MODERNITY } \\
\hline \multicolumn{3}{|l|}{ Household has electricity } \\
\hline No & -- & 1.00 \\
\hline Yes & -- & $1.49(.790,2.81)$ \\
\hline \multicolumn{3}{|l|}{ Household has radio } \\
\hline No & -- & 1.00 \\
\hline Yes & -- & $.657(.376,1.15)$ \\
\hline \multicolumn{3}{|l|}{ Household has TV } \\
\hline No & -- & 1.00 \\
\hline \multirow{2}{*}{\multicolumn{3}{|c|}{$\begin{array}{l}\text { Yes } \\
\text { Household has cable TV }\end{array}$}} \\
\hline & & \\
\hline No & -- & 1.00 \\
\hline Yes & -- & $.234(.056, .972) *$ \\
\hline \multicolumn{3}{|l|}{ Household has Telephone } \\
\hline No & -- & -- \\
\hline Yes & -- & -- \\
\hline \multicolumn{3}{|l|}{ Has mobile telephone } \\
\hline No & -- & 1.00 \\
\hline Yes & -- & $.738(.444,1.23)$ \\
\hline \multicolumn{3}{|c|}{ Multiple exposure to agents of modernity } \\
\hline None & -- & -- \\
\hline One & -- & -- \\
\hline Two & -- & -- \\
\hline More than two & -- & -- \\
\hline \multicolumn{3}{|c|}{ FREQUENCY OF EXPOSURE TO MODERNITY } \\
\hline Frequency listened to radio & & \\
\hline
\end{tabular}




\begin{tabular}{|c|c|c|}
\hline $\begin{array}{l}\text { Not at all } \\
\text { Less than once a week } \\
\text { At least once a week }\end{array}$ & $\begin{array}{l}-- \\
--\end{array}$ & $\begin{array}{l}1.00 \\
.792(.430,1.46) \\
1.32(.691,2.53)\end{array}$ \\
\hline Frequency watch TV & & \\
\hline $\begin{array}{l}\text { Not at all } \\
\text { Less than once a week } \\
\text { At least once a week }\end{array}$ & $\begin{array}{l}-- \\
--\end{array}$ & $\begin{array}{l}1.00 \\
1.52(.785,2.95) \\
.740(.316,1.74) \\
\end{array}$ \\
\hline FERTILITY BEHAVIOUR & & \\
\hline Preference for another child & & \\
\hline $\begin{array}{l}\text { Favorable to another child } \\
\text { Not favorable to another } \\
\text { child }\end{array}$ & $\begin{array}{l}1.00 \\
2.46(1.60,3.79)^{* * * *}\end{array}$ & $\begin{array}{l}1.00 \\
2.41(1.25,4.65)^{* *}\end{array}$ \\
\hline No of living children & & \\
\hline $\begin{array}{l}\text { Three or more } \\
\text { Two or less }\end{array}$ & $\begin{array}{l}1.00 \\
.506(.324, .790) * *\end{array}$ & $\begin{array}{l}1.00 \\
.491(.264, .915) *\end{array}$ \\
\hline $\begin{array}{l}\text { Note: Level of Significance; } \\
\text { likelihood }=\text { I I } 20.834^{\mathrm{a}}, \text { Nagelk } \\
=700.762^{\mathrm{a}}, \text { Nagelkerke R Squ }\end{array}$ & $\begin{array}{l}\mathrm{p}<.05,{ }^{*} \mathrm{p}<.01 \\
\text { rke R Square }=.029 \\
\mathrm{re}=.362(36 \%)\end{array}$ & $\begin{array}{l}\text { odel 3: Chi-square }=30.935,-2 \text { Log } \\
\text { Chi-square }=362.960,-2 \text { Log likelihood }\end{array}$ \\
\hline
\end{tabular}

Table 4, examined the relationships between modern contraceptive use of rural women married/living with partner and predictors treated in four blocks of models with Model I considering only socioeconomic variables, Model 2 treated only agents of modernity, Model 3 dealt with fertility behavior variables, and Model 4 is the full model that included all predictors. This type of modeling enabled teasing out the independent direct effects of socioeconomic, agents of modernity, and fertility behavior on contraceptive use. The strengthen of the relationships with respect to point estimates were tested at three levels, .05 , $.0 \mathrm{I}$, and $.00 \mathrm{I}$, and confidence interval which provided the range for the actual population parameter was set at $95 \%$. Model summary results in Table 4 show that Model 4 is the best fit of the four models with a high Chi-square value (362.96), lowest -2 log likelihood (700.76), and highest explained variance of $36 \%$.

The presentation of results in this section focuses on the full model (Model 4) which considered all predictors at the same time, and showed predictors that had direct and indirect effects on contraceptive use. Comparing significant results in Model 4 with those in Models I-3 show that respondent's state of residence, level of education, religion, wealth status, and number of siblings had direct effects on modern contraceptive use, and husband/partner's education had indirect effects on use. The odds of modern contraceptive use were higher for respondents who lived in rural areas of Zamfara $(\mathrm{OR}=8.95,[\mathrm{Cl} 2.44$, 32.82], $\mathrm{p} \leq .0 \mathrm{I})$, and Kaduna $(\mathrm{OR}=13.45,[\mathrm{Cl} 3.64$, 49.68], $\mathrm{p} \leq .00 \mathrm{I})$ states compared to their counterparts in Sokoto state, the reference category.
Likewise the odds of contraceptive use were higher for rural respondents with primary education than for those with no education $(\mathrm{OR}=2.24,[1.17,4.28], \mathrm{p} \leq$ .05); higher for Christians compared to Muslims $(\mathrm{OR}=10.22$, [4.77, 21.92], $\mathrm{p} \leq .00 \mathrm{I})$; and higher for richer compared to poorest/poorer wealth category $(\mathrm{OR}=6.08,[1.94,18.99], \mathrm{p} \leq .0 \mathrm{I})$. Also, the odds of modern contraceptive use were higher for rural respondents that had six or more siblings $(O R=2.4 \mathrm{I}$, $[1.09,5.28], \mathrm{p} \leq .05)$ and higher for those that had three to five siblings ( $O R=2.23,[1.06,4.67], p \leq .05)$ compared to their counterparts who had two, one or no sibling.

This study examined the effects of agents of modernity on modern contraceptive use among rural respondents in north-west region of Nigeria. Results in Table 4 show that only respondents who had cable TV in their household had direct effects on contraceptive use. It was unexpected that the odds of using modern contraceptive was less $(O R=.234$, [.056, .972], $\mathrm{p} \leq .05)$, for respondents who had cable TV compared to those who reported none. Results on predictors of fertility behaviors show that preference for another child had direct effect on contraceptive use while number of living children had indirect effect. The odds of modern contraceptive use among respondents who lived in the rural areas was higher for those who were not favorable to having another child $(\mathrm{OR}=2.4 \mathrm{I},[\mathrm{I} .25,4.65], \mathrm{p} \leq .0 \mathrm{I})$ compared to their counterparts who wanted another child during the NDHS. 
Key Determinants of Modern Contraceptive Use in Urban Areas

Table 5: The Odds that married women and those in union aged 15 - 49 living in urban areas used modern contraceptives by predictors

\begin{tabular}{|c|c|c|}
\hline & Model 5 & Model 6 \\
\hline Variables & OR $(95 \% \mathrm{Cl})$ & OR $(95 \% \mathrm{Cl})$ \\
\hline \multicolumn{3}{|c|}{ MARRIED WOMEN BACKGROUND VARIABLES } \\
\hline \multicolumn{3}{|c|}{ Age of respondent } \\
\hline (single years) & $1.10(.891,1.37)$ & -- \\
\hline \multicolumn{3}{|l|}{ State of residence } \\
\hline Sokoto & 1.00 & -- \\
\hline Kebbi & $.826(.253,2.69)$ & -- \\
\hline Zamfara & $2.24(.910,5.49)$ & -- \\
\hline Katsina & $1.22(.463,3.19)$ & -- \\
\hline Kaduna & $2.14(.867,5.26)$ & -- \\
\hline Kano & $.242(.088, .666) * *$ & -- \\
\hline Jigawa & $.228(.027,1.92)$ & -- \\
\hline \multicolumn{3}{|l|}{ Highest educational level } \\
\hline No education & 1.00 & -- \\
\hline Primary & $1.86(.891,3.89)$ & -- \\
\hline Secondary or higher & $1.85(.941,3.63)$ & -- \\
\hline \multicolumn{3}{|l|}{ Religion of respondent } \\
\hline Islam & 1.00 & -- \\
\hline Christianity & $3.21(1.63,6.33)$ **** & -- \\
\hline \multicolumn{3}{|l|}{ Wealth index } \\
\hline Poorest- poorer & 1.00 & -- \\
\hline Middle & $1.00(.321,3.12)$ & -- \\
\hline Richer & $2.08(.712,6.05)$ & -- \\
\hline Richest & $3.25(1.07,9.88) *$ & -- \\
\hline \multicolumn{3}{|l|}{ No of co-wives } \\
\hline No other wives & 1.00 & -- \\
\hline One or more & $.928(.530,1.62)$ & -- \\
\hline \multicolumn{3}{|l|}{ Living arrangement } \\
\hline Wife living with husband & 1.00 & -- \\
\hline \multirow[t]{2}{*}{ Wife living elsewhere } & & \\
\hline & $397(.102,1.55)$ & -- \\
\hline \multicolumn{3}{|c|}{ Number of siblings of respondent } \\
\hline None-two or less & 1.00 & -- \\
\hline Three to five & $.568(.298,1.08)$ & -- \\
\hline Six or more & $.760(.368,1.57)$ & -- \\
\hline \multicolumn{3}{|c|}{ Birth order of respondent } \\
\hline First child & 1.00 & -- \\
\hline \multirow{2}{*}{$\begin{array}{l}\text { Second or third child } \\
\text { Forth child or higher }\end{array}$} & $.579(.340, .984) *$ & -- \\
\hline & $1.03(.578,1.85)$ & -- \\
\hline \multicolumn{3}{|l|}{ Employment status } \\
\hline No working & 1.00 & -- \\
\hline Working & $3.84(.765,19.30)$ & -- \\
\hline \multicolumn{3}{|l|}{ Type of employment } \\
\hline Not working- others & 1.00 & -- \\
\hline \multicolumn{3}{|l|}{ Skilled-unskilled manual } \\
\hline Agricultural & $.342(.056,2.10)$ & -- \\
\hline \multirow[t]{3}{*}{ Technical-services } & & \\
\hline & $.000(.000,--)$ & -- \\
\hline & $.545(.102,2.92)$ & -- \\
\hline
\end{tabular}


African Population Studies Vol 33, No. 2, 2019

\begin{tabular}{|c|c|c|}
\hline Not working & -- & -- \\
\hline Cash or kind & -- & -- \\
\hline Cash only & -- & -- \\
\hline \multicolumn{3}{|l|}{ Husband Age } \\
\hline (single years) & $.996(.962,1.03)$ & -- \\
\hline \multicolumn{3}{|c|}{ Husband highest educational level } \\
\hline $\begin{array}{l}\text { No education-don't know } \\
\text { Primary }\end{array}$ & 1.00 & -- \\
\hline Secondary & $.991(.368,2.67)$ & -- \\
\hline Higher & $1.33(.604,2.93)$ & -- \\
\hline & $2.33(1.04,5.25) *$ & -- \\
\hline \multicolumn{3}{|c|}{ Respondents husband type of work } \\
\hline $\begin{array}{l}\text { Skilled-unskilled manual } \\
\text { Agricultural }\end{array}$ & 1.00 & -- \\
\hline Technical services & $\begin{array}{l}.656(.225,1.91) \\
1.07(.640,1.80)\end{array}$ & $\begin{array}{l}-- \\
--\end{array}$ \\
\hline
\end{tabular}

\begin{tabular}{|c|c|c|}
\hline \multicolumn{3}{|l|}{ AGENTS OF MODERNITY } \\
\hline \multicolumn{3}{|l|}{ Household has electricity } \\
\hline No & -- & \multirow{2}{*}{$\begin{array}{l}1.00 \\
1.34(.494,3.64)\end{array}$} \\
\hline Yes & -- & \\
\hline \multicolumn{3}{|l|}{ Household has radio } \\
\hline No & -- & \multirow{2}{*}{$\begin{array}{l}I .00 \\
1.7 I(.660,4.4 I)\end{array}$} \\
\hline Yes & -- & \\
\hline \multicolumn{3}{|l|}{ Household has TV } \\
\hline No & -- & \multirow{2}{*}{$\begin{array}{l}1.00 \\
3.62(1.63,8.06) * *\end{array}$} \\
\hline Yes & -- & \\
\hline \multicolumn{3}{|l|}{ Household has cable TV } \\
\hline No & -- & \multirow{2}{*}{$\begin{array}{l}1.00 \\
1.87(1.24,2.82) * *\end{array}$} \\
\hline Yes & -- & \\
\hline \multicolumn{3}{|l|}{ Household has Telephone } \\
\hline No & -- & 1.00 \\
\hline Yes & -- & $.228(.078, .663) * *$ \\
\hline \multicolumn{3}{|l|}{ Has mobile telephone } \\
\hline No & -- & \multirow{2}{*}{$\begin{array}{l}1.00 \\
10.22(1.37,76.13) *\end{array}$} \\
\hline Yes & -- & \\
\hline \multicolumn{3}{|c|}{ Multiple exposure to agents of modernity } \\
\hline None & -- & -- \\
\hline One & -- & -- \\
\hline Two & -- & -- \\
\hline More than two & -- & -- \\
\hline \multicolumn{3}{|c|}{ FREQUENCY OF EXPOSURE TO MODERNITY } \\
\hline \multicolumn{3}{|c|}{ Frequency listened to radio } \\
\hline Not at all & -- & \multirow{2}{*}{$\begin{array}{l}1.00 \\
2.13(1.04,4.35) *\end{array}$} \\
\hline Less than once a week & -- & \\
\hline At least once a week & -- & $1.15(.563,2.35)$ \\
\hline \multicolumn{3}{|l|}{ Frequency watch TV } \\
\hline Not at all & -- & 1.00 \\
\hline \multirow{2}{*}{$\begin{array}{l}\text { Less than once a week } \\
\text { At least once a week }\end{array}$} & -- & $3.89(1.99,7.59) * * *$ \\
\hline & -- & $2.68(1.36,5.31)^{* *}$ \\
\hline \multicolumn{3}{|l|}{ FERTILITY BEHAVIOUR } \\
\hline Preference for another child & & \\
\hline
\end{tabular}


Favorable to another child

Not favorable to another child

No of living children

Three or more

Two or less

Note: Level of Significance; ${ }^{*} p<.05,{ }^{* *} p<.01$, ${ }^{* * *} p<.001$; Model 5: Chi-square $=297.98 \mathrm{I},-2$ Log likelihood $=651.695^{\mathrm{a}}$, Nagelkerke R Square $=.383(38 \%)$; Model 6: Chi-square $=154.161,-2$ Log likelihood $=827.005^{\mathrm{a}}$, Nagelkerke R Square $=.199(20 \%)$

Table 5: The Odds that married women and those in union aged $15-49$ living in urban areas used modern contraceptives by predictors (continued)

\begin{tabular}{|c|c|c|}
\hline & Model 7 & \multirow{2}{*}{$\begin{array}{l}\text { Model } 8 \\
\text { OR }(95 \% \mathrm{Cl})\end{array}$} \\
\hline Variables & OR $(95 \% \mathrm{Cl})$ & \\
\hline \multicolumn{3}{|c|}{ MARRIED WOMEN BACKGROUND VARIABLES } \\
\hline \multicolumn{3}{|c|}{ Age of respondent } \\
\hline (single years) & -- & $.776(.596,1.01)$ \\
\hline \multicolumn{3}{|l|}{ State of residence } \\
\hline Sokoto & -- & 1.00 \\
\hline Kebbi & -- & $\mathrm{I} .43(.390,5.22)$ \\
\hline Zamfara & -- & $3.42(1.16,10.12) *$ \\
\hline Katsina & -- & $.944(.268,3.32)$ \\
\hline Kaduna & -- & $2.18(.776,6.15)$ \\
\hline Kano & -- & $.323(.104,1.00) *$ \\
\hline Jigawa & -- & $.293(.032,2.66)$ \\
\hline \multicolumn{3}{|l|}{ Highest educational level } \\
\hline No education & -- & 1.00 \\
\hline Primary & -- & $1.92(.863,4.25)$ \\
\hline Secondary or higher & -- & $1.89(.903,3.93)$ \\
\hline \multicolumn{3}{|l|}{ Religion of respondent } \\
\hline Islam & -- & 1.00 \\
\hline Christianity & -- & $3.99(1.89,8.44) * * *$ \\
\hline \multicolumn{3}{|l|}{ Wealth index } \\
\hline Poorest- poorer & -- & 1.00 \\
\hline Middle & -- & $.440(.114,1.69)$ \\
\hline Richer & -- & $.556(.125,2.47)$ \\
\hline Richest & -- & $.727(.151,3.49)$ \\
\hline \multicolumn{3}{|l|}{ No of co-wives } \\
\hline No other wives & -- & 1.00 \\
\hline One or more & -- & $.914(.494,1.69)$ \\
\hline \multicolumn{3}{|l|}{ Living arrangement } \\
\hline \multirow{2}{*}{$\begin{array}{l}\text { Wife living with husband } \\
\text { Wife living elsewhere }\end{array}$} & -- & 1.00 \\
\hline & -- & $202(002,65)$ \\
\hline \multicolumn{3}{|c|}{ Number of siblings of respondent } \\
\hline None-two or less & -- & 1.00 \\
\hline Three to five & -- & $.625(.308,1.27)$ \\
\hline Six or more & -- & $.661(.298,1.47)$ \\
\hline \multicolumn{3}{|c|}{ Birth order of respondent } \\
\hline First child & -- & 1.00 \\
\hline \multirow{2}{*}{$\begin{array}{l}\text { Second or third child } \\
\text { Forth child or higher }\end{array}$} & -- & $.558(.313, .994) *$ \\
\hline & -- & $1.13(.602,2.11)$ \\
\hline \multicolumn{3}{|l|}{ Employment status } \\
\hline No working & -- & 1.00 \\
\hline
\end{tabular}


African Population Studies Vol 33, No. 2, 2019

\begin{tabular}{|c|c|c|}
\hline Working & -- & $1.92(.359,10.27)$ \\
\hline \multicolumn{3}{|l|}{ Type of employment } \\
\hline \multirow{2}{*}{\multicolumn{3}{|c|}{$\begin{array}{l}\text { INot Working- otners } \\
\text { Skilled-unskilled manual }\end{array}$}} \\
\hline & & \\
\hline Agricultural & -- & $.619(.091,4.23)$ \\
\hline \multicolumn{3}{|l|}{ Technical-services } \\
\hline & -- & $.000(.000,--)$ \\
\hline & -- & $1.37(.236,7.92)$ \\
\hline \multicolumn{3}{|c|}{ Earnings from employment } \\
\hline \multirow{3}{*}{\multicolumn{3}{|c|}{$\begin{array}{l}\text { Not working } \\
\text { Cash or kind } \\
\text { Cash only }\end{array}$}} \\
\hline & & \\
\hline & & \\
\hline \multicolumn{3}{|l|}{ Husband Age } \\
\hline (single years) & -- & $.996(.959,1.03)$ \\
\hline \multicolumn{3}{|c|}{ Husband highest educational level } \\
\hline No education-don't know & -- & 1.00 \\
\hline \multicolumn{3}{|l|}{ Primary } \\
\hline Secondary & -- & $.931(.325,2.67)$ \\
\hline \multirow{2}{*}{ Higher } & -- & $1.20(.526,2.75)$ \\
\hline & -- & $2.36(1.00,5.56) *$ \\
\hline \multicolumn{3}{|c|}{ Respondents husband type of work } \\
\hline \multirow{2}{*}{\multicolumn{3}{|c|}{$\begin{array}{l}\text { Skilled-unskilled manual } \\
\text { Agricultural }\end{array}$}} \\
\hline & & \\
\hline \multirow[t]{2}{*}{ Technical services } & -- & $.713(.230,2.20)$ \\
\hline & -- & $.844(.486,1.47)$ \\
\hline \multicolumn{3}{|c|}{ AGENTS OF MODERNITY } \\
\hline \multicolumn{3}{|c|}{ Household has electricity } \\
\hline No & -- & 1.00 \\
\hline & -- & $.626(.172,2.28)$ \\
\hline \multicolumn{3}{|l|}{$\begin{array}{l}\text { Tes } \\
\text { Household has radio }\end{array}$} \\
\hline No & -- & 1.00 \\
\hline \multirow{2}{*}{\multicolumn{3}{|c|}{$\begin{array}{l}\text { Yes } \\
\text { Household has TV }\end{array}$}} \\
\hline & & \\
\hline No & -- & 1.00 \\
\hline \multirow{2}{*}{\multicolumn{3}{|c|}{$\begin{array}{l}\text { Yes } \\
\text { Household has cable TV }\end{array}$}} \\
\hline & & \\
\hline No & -- & 1.00 \\
\hline \multirow{2}{*}{\multicolumn{3}{|c|}{$\begin{array}{l}\text { Yes } \\
\text { Household has Telephone }\end{array}$}} \\
\hline & & \\
\hline No & -- & -- \\
\hline Yes & -- & -- \\
\hline \multicolumn{3}{|l|}{ Has mobile telephone } \\
\hline No & -- & 1.00 \\
\hline Yes & -- & $8.58(1.09,66.93) *$ \\
\hline \multicolumn{3}{|c|}{ Multiple exposure to agents of modernity } \\
\hline None & -- & -- \\
\hline One & -- & -- \\
\hline Two & -- & -- \\
\hline More than two & -- & -- \\
\hline \multicolumn{3}{|c|}{ FREQUENCY OF EXPOSURE TO MODERNITY } \\
\hline Frequency listened to ra & & \\
\hline Not at all & -- & 1.00 \\
\hline Less than once a week & -- & $1.26(.525,3.01)$ \\
\hline At least once a week & -- & $1.52(.623,3.72)$ \\
\hline
\end{tabular}




\begin{tabular}{|c|c|c|}
\hline \multicolumn{3}{|l|}{ Frequency watch TV } \\
\hline Not at all & -- & 1.00 \\
\hline Less than once a week & -- & $2.39(1.02,5.59) *$ \\
\hline \multicolumn{3}{|l|}{ At least once a week } \\
\hline \multicolumn{3}{|l|}{ FERTILITY BEHAVIOUR } \\
\hline \multicolumn{3}{|l|}{ Preference for another child } \\
\hline Favorable to another child & 1.00 & 1.00 \\
\hline Not favorable to another child & $2.48(1.67,3.68)$ **** & $3.30(1.74,6.27)$ **** \\
\hline \multicolumn{3}{|l|}{ No of living children } \\
\hline Three or more & 1.00 & 1.00 \\
\hline Two or less & $.780(.526,1.16)$ & $.398(.226, .702) * * *$ \\
\hline \multicolumn{3}{|c|}{$\begin{array}{l}\text { Note: Level of Significance; }{ }^{*} p<.05,{ }^{*} \mathrm{p}<<.01,{ }^{*} * * *{ }^{*}<.00 I ; \text { Model 7: Chi-square }=24.166,-2 \text { Log } \\
\text { likelihood }=970.223^{\mathrm{a}}, \text { Nagelkerke R Square }=.032(3.2 \%) ; \text { Model 8: Chi-square }=346.206,-2 \text { Log likelihood } \\
=565.646^{\mathrm{a}}, \text { Nagelkerke R Square }=.453(45 \%)\end{array}$} \\
\hline
\end{tabular}

Table 5 above examined the relationships between modern contraceptive use among urban women married/living with spouse and predictors. The data analysis followed the same pattern of block modeling employed for data analysis on rural respondents. Analysis tested the strengthen of the relationships in terms of point estimates significant levels at $.05, .01$, and .001 , and confidence interval was set at $95 \%$. Results of Models 5 to 8 summarized in Table 5 show that Model 8 is the best fit of the four models considered in this section with the highest Chi-square value (346.20), lowest -2 log likelihood (565.65), and highest explained variance of $45 \%$.

As with the rural respondents results in this section focus was on the full model (Model 8) which considered all predictors simultaneously. Comparing significant results in Model 8 with those in Models 5-7 show that respondent's socioeconomic factors that had direct effects on modern contraceptive use were state of residence, religion, birth order of respondent, and husband/partner's education, while wealth index had indirect effects on use. The odds of modern contraceptive use were higher for respondents who lived in urban areas of Zamfara state $(\mathrm{OR}=3.42,[\mathrm{Cl} I .16,10.12], \mathrm{p} \leq .05)$, and lower for those in Kano state $(\mathrm{OR}=.32,[\mathrm{Cl} .10, \mathrm{I} .00], \mathrm{p} \leq$ .05) compared to their counterparts in Sokoto state. Also, the odds of contraceptive use were higher for urban respondents who were Christians $(\mathrm{OR}=3.99$, [Cl I.89, 8.44], $\mathrm{p} \leq .00 \mathrm{I}$ ) compared to their Muslims counterparts. The odds of contraceptive use were lower for respondents who were second or third child $(\mathrm{OR}=.558,[\mathrm{Cl} .3 \mathrm{I}, .99], \mathrm{p} \leq .05)$ compared to those who were first child. The odds of contraceptive use were higher for those whose husbands/partners had a higher education $(\mathrm{OR}=2.36,[\mathrm{Cl} \mathrm{I} .00,5.56], \mathrm{P}$ $\leq .05)$ than their counterparts whose husband/partner had no education/don't know. And results showed that birth order of respondent had 4995 indirect effects on modern contraceptive use in urban areas of North-West region of Nigeria.

In the urban areas, results showed that agents of modernity that had direct effects on modern contraceptive use were; has radio in household, has mobile telephone, and frequency watched TV, and those with indirect effects were household has; TV, cable TV, and frequency listened to radio. Results in Table 5 show that respondents in urban areas who had TV in their household had higher odds $(\mathrm{OR}=3.57,[\mathrm{Cl} 1.09,1 \mathrm{I} .73], \mathrm{p} \leq .05)$ of modern contraceptive use compared to their counterpart who had no TV. Also, the odds of contraceptive use were higher for respondents who had mobile telephone $(\mathrm{OR}=8.58,[\mathrm{Cl}$ I.09, 66.93], $\mathrm{p} \leq .05)$ compared to those who reported none. Respondents who watched TV less than once a week had higher odds of contraceptive use $(\mathrm{OR}=2.39,[\mathrm{Cl} I .02,5.59]$, $\mathrm{p} \leq .05)$ compared to their contemporaries who did not watch TV, while those who watched TV at least once a week had indirect effect on contraceptive use.

Results on fertility behaviors suggest that both preference for another child and number of living children had direct effects on modern contraceptive use among respondents in the urban areas. The odds of modern contraceptive use was higher among respondents who were not favorable to have another child $(O R=3.30,[I .74,6.27], p \leq .00 I)$ compared to their counterparts who were favorable, and the odds of contraceptive use were lower for respondents who had two/one/no children compared to those who had three or more during the NDHS.

\section{Discusssions}

This paper examined key factors affecting contraceptive use in Northwestern region of Nigeria with the aim of providing policy and program relevant information to increase usage. Key factors examined include background characteristics of individual 
women aged 15-49 married/living with partner, their husbands' selected background factors, agents of modernity, and fertility behavior factors. The paper analyzed a sub-sample of 8319 married women extracted from the national sample of the NDHS. Analysis and findings disaggregated between urban and rural respondents to provide more insights on the factors influencing modern contraceptive use in the region.

The findings showed significant differences in factors influencing modern contraceptive use among residents in rural vs. urban areas of the northwestern, which are unique insights provided by this study, and are not readily available in the literature. The first key observation is that more background factors were significant in rural than in urban areas suggesting that future family planning programming should be more residence specific to increase effectiveness. Two background factors, state of residence and religion serve as common denominators having significant direct relationships with modern contraceptive usage both in rural and urban areas. The importance of these two variables need to be factored into programming to improve contraceptive uptake in the region. Although significant variations were not uniform across all states, the findings suggest significant differentials in modern contraceptive use in Zamfara and Kaduna compared to Sokoto in the rural areas, and Zamfara and Kano compared to Sokoto in the urban areas. These differentials in contraceptive use may be along ethnic or cultural lines. It will be necessary that further studies examine state differences in modern contraceptive use along ethnic or cultural differences with a view to providing additional insights for family planning programming to increase uptake in the states.

Findings of this study confirm the importance of religion in contraceptive use in the region (Konkor, et al., 2019; Zaggi, 20l4; Muhammad and Maimuna, 2014; Olaitan, 20II). Religious influences in contraceptive use cuts across rural vs. urban residence, with findings showing higher odds for contraceptive use among Christians than Muslims in the region (Konkor, et al., 2019). Thus, indicative of the facts that approaches to increase contraceptive use will have to factor-in strategies for eliciting the cooperation of the Muslim sub-groups who are in the majority in the region. Also, since religion is a common denominator in modern contraceptive use in both rural and urban areas, programming to change the behavior of respondents who lived in Muslim dominated areas need to be different from that implemented among their Christian counterparts to elicit the expected or desired effect.
This study reinforces evidence in the literature on the positive relationship between contraceptive use and women's education (Konkor, et al, 2019; Igbodekwe et al, 20I4; Asekun-Olarinmoye et al, 20I3; Olalekan and Olufunmilayo, 2012) especially primary school education in the rural areas of the north-west region. This probably is because education enables women to better appreciate the benefits of family planning during exposure to messages and counselling sessions on anti-natal visits where this is done (AbekahNkrumah and Abor, 2016). The importance of husband/partner's education was evident in the urban areas where there were higher odds of respondents' contraceptive use compared with those whose husband/partner were uneducated, which reinforcing the need to gain more attention of uneducated (Abekah-Nkrumah and Abor, 2016). Women education in the rural areas, and men's education in the urban areas can be a two-pronged strategy that may be explored by the government and interested stakeholders in the short-term and long-term to increase chances of modern contraceptive use in the region.

A unique finding for only rural respondents was that the odds of modern contraceptive use was higher for those of richer wealth status than for respondents in the poorer/poorest sub-groups (Konkor et al., 2019; Abekah-Nkrumah and Abor, 2016). This significant difference may be linked to accessibility challenges that rural respondents faced probably due to distance and/or cost of family planning services (Odewale et al, 2016; Olaitan, $20 \mathrm{II}$ ). Since health care facilities are less accessible in most rural areas, respondents of a higher wealth status are more likely able to pay for cost attributable to distance to family planning facility than their rural counterparts if they are well motivated. Since mass media using agents of modernity is an effective medium for family planning information (Konkor, et al, 2019; Rogers, 2018; Owoloko and Oladosun, 20I8), further studies is needed to tease out effects or impact of accessibility challenges in demand creation for modern contraceptive use in this region of the country, and especially as it differs across the states.

Another unique finding in the rural areas of the north-west region is the significant relationship between contraceptive use and respondent's number of siblings. The odds of contraceptive use were higher for respondents from large family compared to their counterparts from small family. The reasons may be that those who had many siblings experienced negative consequences of large family size and do not what the same experience in their own immediate family. This area will be interesting for further study to examine linkages between 
number of siblings, fertility behavior and modern contraceptive use.

This study corroborated another study which found a significant relationship between modern contraceptive use and birth order (Abekah-Nkrumah and Abor, 2016). It found that the odds of contraceptive use were lower for urban respondents who were of the second and third birth order compared to those who were first born. Again, the reason may be that respondents who were first born had more responsibilities taking care of younger siblings thus, would rather use modern contraceptive to limit their desired family than have large family size.

In general, agents of modernity, except possession of cable TV, were not significant predictors of contraceptive use in the rural areas. These results were not unexpected because modernity agents such as TV, cable TV, and even telephone are far less available or inaccessible in the rural areas of Nigeria as a whole (north-west inclusive). The finding that respondents in the rural areas who had cable TV had lower odds of contraceptive use was quite unexpected since cable TV is a veritable mass media tool for accessing family planning information (Konkor et al, 2019; Rogers, 2018). The reason for this result may be that the cable TV was not engaged often or used sparingly for entertainment purposes only since access to electricity is also poor in most rural areas in the country. Further studies should examine ways of improving access to agents of modernity in the rural areas of the north-west region since these are adjudged an efficient means of effective contraceptive social marketing in the country (Knotor et al, 2019; Ugboaja et al, 2018).

As expected, possession of agents of modernity such as radio, and mobile phone had positive effects on modern contraceptive use in the urban areas. Radio and mobile phone can become invaluable agents of modernity for exchange of vital family planning information in Nigeria if customized to address needs in specific sub-groups of the population with the aim of substantial uptake in modern contraceptive use (Knotor et al, 2019; Ugboaja et al, 2018; Silvast and Mikko, 2014; Kyem, 2012). Another expected result of this study is that contraceptive use is positively related to frequency of watching TV, an indicator of effective use of agents of modernity (Ugboaja et al, 2018; Kyem, 20I2; Kyem and LeMaire, 2006). The result confirms the evidence that population and family planning policies and program that use TV platforms, as change medium will achieve the desired results in the region (Rogers, 2018; Ugboaja et al, 2018).

Evidence in the literature suggest that future fertility limiting behaviors are more favorable to contraceptive use (Unumeri et al, 20I5; Asiimwe et al, 20।4; Frini et al, 20।3; Arbab et al, 20I I; Olaitan, $201 \mathrm{I}$; Oyediran and Isiugo-Abanihe, 2002). Evidence in this study suggest that the north-west region is already 'pregnant' with the demand for fertility limitation especially with the odds of modern contraceptive use higher for rural and urban respondents that were not favorable to having another child (Oladosun et al, 2019), and lower for urban respondents who had more living children than those who had fewer or none (Oladosun et al, 2019; Abekah-Nkrumah et al, 2016) despite high awareness about modern contraceptive methods (NPC [Nigeria] and ICF International, 20I4). Family planning programs should make these favorable contraceptive use behaviors more widespread and acceptable in the region. In order to increase uptake of modern contraceptive in the region, it may be necessary to examine the characteristics of urban and rural respondents not favorable to having another child and were not using any method. This type of segmentation analysis will provide more information that can be used to customize campaign that can serve as catalyst to increase demand and use of modern contraceptives in the region.

\section{Conclusions}

Key factors significant to modern contraceptive use irrespective of area of residence are state of residence, religion, and preference for another child. These three common denominators should be prime in program intervention geared to increase modern contraceptive uptake in the north-west region of Nigeria. This study filled gaps in the literature by using disaggregated analysis to provide insights on specific predictors common to both rural and urban areas, and those unique to each of the areas. In addition to these common denominators, effective customized programming should consider education, wealth status, respondent's number of siblings, and have cable TV for the rural areas of the region. Unique predictors for urban areas programming include respondent's birth order, husband's education, and number of living children. In order to reduce fertility and increase contraceptive use, policies and programs need to incorporate these key unique factors into programming to achieve the desired result of modern contraceptive uptake and fertility reduction. The tasks of family planning policies and programs is to make the nascent fertility limitation behavior evident among some respondents in this study area, and cascade to the larger population by custom-tailored demand creation strategies to make it more socially acceptable for couples to transition from large to small family size 
among communities in the north-west sub-region of Nigeria.

\section{Comflicting interests}

The authors take this opportunity to declare that there are no competing interests in the writing and submission of this paper for publication.

\section{Acknowledgement}

We express our gratitude to Covenant University, Ota, Nigeria for funding the publication of this article. In addition, we thank DHS Program, Rockville, MD, USA for making the 2013 NDHS women individual data available for use.

\section{Authors' contributions}

Muyiwa Oladosun conceptualized the topic of study while Tayo George and Anthony Onwumah prepared the literature review with contributions from Adebanke Olawale-Isaac and Dorcas Adekoya. Muyiwa Oladosun did the data analysis, and drafted the sections on results and discussion. All authors reviewed and provided suggestions on the final draft.

\section{References}

Abekah-Nkrumah G and Aseweh A (2016). Socioeconomic determinants of use of reproductive health services in Ghana. Health Economic Review; 6:9.

Anyebe EE, Olufemi SK, and Lawal HRN (2014). Contraceptive Use among Married Women in Zaria, Northwest Nigeria. Research on Humanities and Social Sciences, 2014. www.iiste.org ISSN (Paper)2224-5766 ISSN (Online)2225-0484 (Online) Vol.4, No.27, 2014

Arbab AA, Bener A and Abdulmalik $M$ (201I). Prevalence, awareness and determinants of contraceptive use in Qatari women. East Mediterranean Health Journal, I7(I): II-I8.

Asekun-Olarinmoye E, Adebimpe WO, Bamidele JO, Odu O, Asekun- Olarinmoye $1 \mathrm{O}$ and Ojofeitimi EO (20|3). Barriers to use of modern contraceptives among women in an inner city area of Osogbo metropolis, Osun State, Nigeria International Journal of Women's Health; (5): 647-655.

Asiimwe, B.J., Ndugga, P., Mushomi, J., Ntozi, J.P.M. (20/4). Factors associated with modern contraceptive use among young and older women in Uganda; a comparative analysis. BMC Public Health, 14:926

Austine, A. (2015). Unmet contraceptive need among married Nigerian women: an examination of trends and drivers. Journal of Contraception; 91, $31-38$
Babalola, S., John, N., Ajao B and Speizer IS (20I5). Ideation and Intention to use Contraceptives in Kenya and Nigeria. Journal of Population Studies; 33(8): $211-238$.

Bosveld, W. (1998). Explaining Between Country Variation in Fertility: The Theoretical link between individual behaviour and Social Context Amsterdam Post doctorate OnderZeoekersopleiding Demography Paper; 4, page 17.

Dormann, C.F., Elith, J., Bacher, S., Buchmann, C., Carl, G., Carre, G., Marquez, J.R.G., Gruber, B., Lafourcade, B., Leitao, P.J., Munkemuller, T., Mclean, C., Osborne, P.E., Reineking, B., Schroder, B., Skidmore, A.K., Zurell, D., and Lautenbach, S. (20/3). Collinearity: a review of methods to deal with it and a simulation study evaluating their performance. Ecography; 36: 027046.

Frini, O., Hanena, W.O., Nabag, M., (20I3). The Knowledge and determinant factors of contraceptive use among married Sudanese Women. Applied Science Reports; 4(3): 247-25I.

Gertner, N. (1999). Interference and Reproductive Choice. In Coha, S. and Tanb, N. (Eds.) Reproductive Laws for the 1990's. Contemporary Issues in Biomedicine, Ethics and Society.

Gueye, A., Speizer, I.S., Corroon, M., and Okigbo, C.C. (20/5). Belief in Family Planning Myths at the Individual and Community Levels and Contraceptive Use in Urban Africa. International Perspective on Sexual and Reproductive Health; 4I(4): $191-199$.

Ibisomi, L. (20/4). Is Age Difference between Partners Associated with Contraceptive Use among Married Couples in Nigeria? International Perspectives on Sexual \& Reproductive Health; 40(I): 39 .

Igbodekwe, F.C., Oladimeji, O., Oladimeji, K.E., Adeoye, I.A., Akpa, O.M., and Lawson, L. (20I4). Utilisation of Modern Contraceptive among Women of Childbearing Age in Resource Constraint Setting: Evidence from 2008 National Demographic and Health Survey in Nigeria. Journal of Health Science; 4(3): 72-78.

Konkor, I., Sano, Y., Antabe, R., Kansanga, M., and Luginaah, I. (2019). Exposure to mass media family planning messages among post-delivery women in Nigeria: testing the structural influence model of health communication. The European Journal of Contraception and Reproductive Health Care; 24 (I), 18-23.

Kyem, P.A.K., and LeMaire, P.K. (2006). Transforming recent gains in the digital divide into digital opportunities: Africa and the boom in mobile phone subscription. Electronic Journal of 
Information Systems in Developing Countries; 289 (Special

Issue),

http://www.ejisdc.org/ojs2/index.php/ejisdc/article /view/343.

Kyem, P.A.K. (20I2). Is ICT the panacea to subSaharan Africa's development problems? Rethinking Africa's contentious engagement with the global information society. Progress in Development Studies; 12 (2\&3): 23 I- 244.

Muhammad, Z., and Maimuna, D.G. (20/4). Contraceptive trend in a tertiary facility In North Western Nigeria: A 10-year review. Department of Obstetrics and Gynaecology, Bayero University, Aminu Kano Teaching Hospital, Kano, Nigeria. [Downloaded free from http://www.njbcs.net on Thursday, August 3, 20I7, IP: 80.248.0.226]

National Bureau of Statistics (NBS) (2018). Demographic Statistics Bulletin 2017. Federal Republic of Nigeria.

National Population Commission (NPC) [Nigeria] and ORC Macro (2004). Nigeria Demographic and Health Survey 2003. Calverton, Maryland: National Population Commission and ORC Macro.

National Population Commission (NPC) [Nigeria] and ICF International (20I4). Nigeria Demographic and Health Survey 2013. Abuja, Nigeria, and Rockville, Maryland, USA: NPC and ICF International.

Ochako, R., Mbondo, M., Aloo, S., Kaimenyi, S., Thompson, R., Temmerman, M., and Kays, M. (2015). Barriers to modern contraceptive methods uptake among young women in Kenya: a qualitative study. BMC Public Health; I5: I 8.

Odewale, J.B., Oladosun, M. and Amoo, E.O. (20I6). Fertility Desire and Contraceptive Use among Women in Nigeria. 3rd International Conference on African Development Issues; ISSN:2449-075X. Covenant University.

Odimegwu, O.C. (1999). Family Planning Attitudes and Use in Nigeria: A Factor Analysis. International family planning perspectives; 25 .

Oladosun, M., Akanbi, M., Fasina, F., and Samuel O. (2019). Key predictors of modern contraceptive use among women in marital relationship in South-West region of Nigeria. Int J of Reprod Contracept Obstet Gynecol; 8(7): 2638-2646.

Olaitan, O.L., (20I I). Factors Influencing the Choice of Family Planning among Couples in Southwest, Nigeria. International Journal of Medicine and Medical Sciences; 3(7): 227-232.

Olalekan, A.W., and Olufunmilayo, A.E. (20I2). A Comprehensive Study of Contraceptive use among Rural and Urban Women in Osun State, Nigeria. International Journal of Tropical Disease and Health; 2(3): 2I 4-224.
Ouma, S., Turyasima, M., Acca, H., Nabbale, F., Obita, K.O., Rama, M., Adong, C.C., Openy, A., Beatrice, M.O., Odongo-Aginya, E.I., and Awor, S. (2015). Obstacles to Family Planning in Atiak Health Center IV, Amuru District, Norther Uganda. East Afr Med J; 92(8): 394-400.

Owoloko, I., and Oladosun, M., (2018). Key Determinants of Contraceptive Use Among Married Women in North-Eastern Region of Nigeria. Proceedings of the International Conference on Global and Emerging Trends (ICGET), 2-4 May, 2018.

Oyediran, A.K., and Isiugo-Abanihe, U.C. (2002). Husband-Wife Communication and Couple's Fertility Desires among Yoruba of Nigeria. African Population Studies; 17, (2): 6I-80.

Potter, J.E. (1999). The Persistence of Outmoded Contraceptive Regimes: The Cases of Mexico and Brazil. Population Development; 25(4): 703-739.

Rogers, D. (2018). The Impact of Mass MediaDelivered Family Planning Campaigns in Developing Countries: A meta-analysis. PhD Dissertation, University of Connecticut, USA.

Rogers, E.M., and Kincaid, D.L. (2000). Communication Networks: Towards a new Paradigm for research New York Academic Press; 295-339.

Rutstein, S.O., and Rojas, G. (2006). Guide to DHS Statistics. Demographic and Health Surveys, ORC Macro, Calverton, Maryland USA, September.

Silvast, A., and Mikko, J.V. (20I4). Keeping Systems at Work: Electricity Infrastructure from Control Rooms to Household Practices. Science \& Technology Studies; 27, (2), 93-I I 4.

Thomas, E.M., and Tsovinar, L.H. (2006). Contraceptive Practices in Armenia: Panel Evaluation of an Information -EducationCommunication Campaign. Journal of Social Science and Medicine; 63, 2770-2783.

Ugboaja, J.O., Oguejiofor, C.B., Oranu, E.O., and lgwegbe, A.O. (20I8). Assessing the Influence of Mass Media on Contraceptive Use in Nigeria: A Secondary Analysis of 2013 Nigerian National Demographic and Health Survey. The Nigerian Journal of General Practice; 16, (2): 39-44.

Unumeri, G., Ishaku, S., Ahonsi, B., and Oginni, A. (20I5). Contraceptive Use and Its Socioeconomic Determinants among women in NorthEast and North-West regions of Nigeria: A Comparative Analysis. African Population Studies; 29, (2): $|85|-\mid 867$.

United Nations (UN), Department of Economic and Social Affairs, Population Division (2019). World Population Prospects 2019: Data Booklets (ST/ESA/SER.A/424). Valente, W., and Thomas, W.P.S. (200I). Campaign Exposure and 
African Population Studies Vol 33, No. 2, 2019

Interpersonal Communication as Factors in Contraceptive Use in Bolivia. Journal of Health Communication; 6, 303-322.

Zaggi, Y.H. (20|4). Contraceptive Knowledge and Practices among Students in Federal Polytechnic Kaduna, Nigeria: An Exploratory Study. Thesis presented in fulfillment of the requirements for the degree of Master of Arts (Sociology) in the Faculty of Arts and Social Sciences at Stellenbosch University December, 20I4. 